\title{
Supply Chain Competition: A Market Game Approach
}

\author{
C. Gizem Korpeoglu \\ Department of Industrial Engineering, Bilkent University, 06800, Ankara, Turkey, gizem.korpeoglu@bilkent.edu.tr \\ Ersin Körpeoğlu \\ School of Management, University College London, London, UK, E14 5AA, e.korpeoglu@ucl.ac.uk \\ Soo-Haeng Cho \\ Tepper School of Business, Carnegie Mellon University, Pittsburgh, PA 15213, soohaeng@andrew.cmu.edu
}

\section{Forthcoming at Management Science}

\begin{abstract}
We study supply chains where multiple suppliers sell to multiple retailers through a wholesale market. In practice, we often observe that both suppliers and retailers tend to influence the wholesale market price retailers pay to suppliers. However, existing models of supply chain competition do not capture retailers' influence on the wholesale price (i.e., buyer power), and show that the wholesale price and the order quantity per retailer do not change with the number of retailers. To overcome this limitation, we develop a competition model based on the market-game mechanism in which the wholesale price is determined based on both suppliers' and retailers' decisions. When taking into account retailers' buyer power, we obtain the result that is consistent with the observed practice: as the number of retailers increases, each retailer's buyer power decreases, and each retailer is willing to pay more for her order, so the wholesale price increases. In this case, supply chain expansion to include more retailers (or suppliers) turns out to be more beneficial in terms of supply chain efficiency than what the prior literature shows without considering buyer power. Finally, we analyze the integration of two local supply chains, and show that, although the profit of the integrated supply chain is greater than the sum of total profits of local supply chains, integration may reduce the total profit of firms in a retailer-oriented supply chain that has more retailers than suppliers.
\end{abstract}

Key words: Competition, Game Theory, Supply Chain Management.

\section{Introduction}

The last two decades have witnessed a radical movement from centrally-managed supply chains towards globally decentralized supply chains (Tang 2016). With advancements in information technologies and reduced barriers in global trade, supply chains are expanding so that retailers can buy products from a larger collection of suppliers, and suppliers can sell to a larger number of retailers in various regions of the world (Malhotra et al. 2007, Netessine 2009). These factors transform local supply chains where only a handful of firms transact into integrated supply chains where a multitude of firms transact. When more firms are interlinked, each firm's ability to influence prices (hereafter, market power) changes (Porter 1980). In this paper, we develop a novel competition 
model that captures market power of firms in supply chains, and analyze the impact of supply chain expansion and integration on prices, quantities, and profits of firms and supply chains. ${ }^{1}$

Porter (1980) identifies buyer power, seller power, competition among firms (industry rivalry), and new entrants as vital forces of competition. ${ }^{2}$ Yet, existing models of supply chain competition are able to capture only some of these forces. Corbett and Karmarkar (2001) come closest to capturing these forces as they pose a research question: "what is the effect of "buyer power" or "supplier power" on prices, quantities, and profits for contiguous and for noncontiguous tiers in the supply chain?" (page 967). Although their model which extends the Cournot model to the supply chain setting successfully captures suppliers' seller power, it assumes that retailers are price takers, having no influence on the wholesale price that retailers pay to suppliers. However, in practice, a retailer often possesses buyer power and influences the wholesale price. For example, the Organization for Economic Co-operation and Development (OECD) (2008, page 9) discusses buyer power as follows: "Buyer power is concerned with how downstream firms can affect the terms of trade with upstream suppliers ... A firm has monopsony power if its share of purchases in the upstream input market is sufficiently large that it can cause the market price to fall by purchasing less and cause it to rise by purchasing more ... buyer power result $[\mathrm{s}]$ in lower prices, though the lower price obtained from monopsony power is achieved through the act of purchasing less ..." Similarly, OECD (1998) discusses buyer power as "the ability of a buyer to influence the terms and conditions on which it purchases goods ... Such a buyer would have a profit incentive to reduce its purchases in order to reduce the market price of goods purchased" (pages 18, 19). Thus, retailers' buyer power is not only intuitively appealing, but also corroborated by practitioner reports.

In order to fill this gap between the extant literature and the observed practice, we propose a model that captures buyer power, seller power, competition among firms, and new entrants in a two-tier supply chain. We build on the market-game mechanism introduced by Shapley and Shubik (1977) to model a wholesale market where multiple suppliers sell to multiple retailers. A supplier may cause the wholesale price to change by changing his production decision, and a retailer may cause the wholesale price to change by changing her procurement decision (i.e., retailers exercise buyer power as discussed in OECD (2008)). Because both suppliers and retailers influence the wholesale price, our model captures both suppliers' seller power and retailers' buyer power.

Using our market-game model, we answer the following research questions: (Q1) How does supply chain expansion to include more suppliers or more retailers and the resulting change in buyer power and seller power affect prices and quantities in a supply chain with multiple suppliers and

\footnotetext{
${ }^{1}$ Throughout the paper, supply chain expansion means that either a new supplier or a new retailer enters the supply chain, while supply chain integration means that local supply chains integrate into an aggregate supply chain.

${ }^{2}$ In addition to the forces listed here, Porter (1980) identifies substitutes, which we do not consider in this paper.
} 
retailers? (Q2) How does expansion affect profits and efficiency in a supply chain? (Q3) How does the integration of local supply chains affect the profit of each supplier and each retailer, and the total profit of firms in each local supply chain as well as the profit of the integrated supply chain?

Regarding our first question, our analysis shows that having a larger number of retailers raises the wholesale price and order quantities. This is because as the number of retailers increases, each retailer's buyer power decreases, and each retailer is willing to pay more for her order, so the wholesale price increases. The increase in the wholesale price induces suppliers to produce more, so retailers receive larger order quantities. These results are consistent with OECD (2008), which discusses that prices and quantities decrease with buyer power, ${ }^{3}$ yet they cannot be shown with the model which extends the Cournot model to the supply chain setting (in short, "the Cournot model" for the rest of the paper, while we refer to the model of Cournot 1838 as "the original Cournot model"). Thus, a model that captures buyer power along with other forces of competition is crucial in studying supply chains where multiple suppliers and multiple retailers compete.

With respect to our second research question, we generate the following insights. First, we show that having more suppliers in a supply chain raises the retailer profit and reduces the supplier profit because of more intense competition among suppliers. We also show that, as expected, having more retailers in a supply chain increases the supplier profit due to a higher wholesale price and larger order quantities. More interestingly, having more retailers in a supply chain can increase the retailer profit, especially when the number of suppliers is large. This is because the positive impact of a larger order quantity outweighs the negative impact of a higher wholesale price. Second, we show that the supply chain efficiency is increasing and concave in the number of suppliers and retailers. We also consider entry and exit decisions of firms, and show that reducing entry barriers facilitates the supply chain expansion. These results together suggest that reducing barriers for supply chain expansion is beneficial, especially to small supply chains. Such benefit in the supply chain efficiency is underestimated if retailers' buyer power is not taken into consideration.

To answer our third research question, we study the integration of two local supply chains that causes the number of suppliers and retailers to increase simultaneously. We show that when transaction costs (e.g., due to tariffs or taxes) between two local supply chains decrease sufficiently, suppliers of each supply chain start transacting with retailers of the other supply chain, leading to an integrated supply chain. Because this integration raises the number of both suppliers and retailers, whether it benefits each local supply chain is not immediately clear. We show that this depends on whether these local supply chains are supplier-oriented (i.e., have more suppliers than retailers) or retailer-oriented (i.e., have more retailers than suppliers). Specifically, if both local

${ }^{3}$ Consistently, Shea (1993) empirically shows that prices and quantities in the wholesale market move in the same direction in response to demand side effects by using data from 26 manufacturing industries in the United States. 
supply chains are retailer-oriented or both are supplier-oriented, their integration raises the total profit of firms in both local supply chains. Otherwise, the total profit of firms in a more retaileroriented supply chain (than the other supply chain) may decrease after integration.

\section{Related Literature}

This paper contributes to the literature that analyzes market power and competition in decentralized supply chains. We can categorize this literature into three streams.

The first stream focuses on seller power and buyer power in a supply chain using the Nash bargaining model (e.g., Bernstein and Nagarajan 2012, Kuo et al. 2013, Feng and Lu 2013, Nakkas and $\mathrm{Xu}$ 2018, Chu et al. 2018, and references therein). The standard approach introduced by Nash (1950) assumes a fixed total profit that does not depend on bargaining outcomes, and this profit is split between buyers and sellers based on their bargaining power. As this model focuses on the impact of bargaining power, it does not give an explicit characterization of prices or quantities, nor does it capture firms' entry and exit decisions. Traditional bargaining models also focus on a single buyer and a singe seller, but there are some recent papers that analyze the impact of multiple buyers or sellers. For example, Nagarajan and Bassok (2008) adopt this approach when studying a setting with multiple sellers (suppliers) and a single buyer (assembler) to analyze the impact of the number of suppliers on bargaining outcomes. There are also papers that assume cooperation among independent firms to relax the assumption that the supply chain profit is fixed or to more explicitly characterize prices or quantities (e.g., Lovejoy 2010, Feng and Lu 2012). ${ }^{4}$ Yet, unlike competition models such as the Cournot model or the market game, the focus of these models is on the bargaining mechanism between negotiating parties on two sides to reach an agreement rather than industry-wide competition. In particular, our market game differs from these bargaining models on two fundamental aspects. First, our model does not assume a fixed supply chain profit, and it explicitly characterizes equilibrium prices and quantities. Second, our model analyzes competition among multiple suppliers and retailers, and captures firms' entry and exit decisions without assuming cooperation among independent firms.

The second stream employs a competition model to study supply chains with one supplier and multiple competing retailers (e.g., Lee and Whang 2002, Bernstein and Federgruen 2003, Netessine

\footnotetext{
${ }^{4}$ A more recent development in the Nash bargaining literature is the introduction of Nash-in-Nash bargaining solutions which tackle bilateral oligopoly (e.g., Feng and Lu 2013, Collard-Wexler et al. 2019). "Yet the Nash-in-Nash solution has been criticized by some as an ad hoc solution that nests a cooperative game theory concept of Nash bargaining within a non-cooperative Nash equilibrium." (Collard-Wexler et al. 2019, page 165). Although there are some recent papers that try to provide a non-cooperative foundation for Nash-in-Nash solution, they either assume that each firm conducts its bilateral negotiations independently (i.e., taking the outcome of its bilateral negotiations with other firms as given) at any point in time (e.g., Crawford and Yorukoglu 2012) or extend the Rubinstein (1982) protocol by assuming that time between offers among negotiating firms is infinitely small (e.g., Collard-Wexler et al. 2019).
} 
and Zhang 2005, Bernstein and Federgruen 2005) or supply chains with multiple competing suppliers and one retailer (e.g., Perakis and Roels 2007, Cachon and Kok 2010, Adida et al. 2015). ${ }^{5}$ These papers capture either supplier competition or retailer competition, but they do not capture simultaneous competition among multiple suppliers and multiple retailers, nor do they capture the joint impact of suppliers' seller power and retailers' buyer power. They are also unable to study supply chain integration because this analysis requires an increase in the number of suppliers and retailers simultaneously in a setting where both multiple suppliers and multiple retailers compete.

As the market game is a competition model, it is not suitable for settings with a single supplier or a single retailer (or both), but it is appropriate for relatively understudied settings with multiple suppliers and multiple retailers where no single firm is powerful enough to impose a contract or dictate a wholesale price. Thus, the third and the most relevant stream of literature studies supply chain competition among multiple suppliers and multiple retailers. The closest studies to our paper are Corbett and Karmarkar (2001) (hereafter C\&K) and Adida and DeMiguel (2011) (hereafter A\&D). C\&K consider supply chains where multiple suppliers engage in Cournot competition in the wholesale market and multiple retailers engage in Cournot competition in the retail market under linear deterministic demand. ${ }^{6}$ A\&D build on the $\mathrm{C} \& \mathrm{~K}$ model by incorporating retailer risk aversion, price uncertainty, and product and retailer differentiation. ${ }^{7}$ There are a few other papers that study supply chains with multiple suppliers and multiple retailers. Bernstein and de Vericourt (2008) consider two suppliers competing to sign procurement contracts with two retailers. Cho (2014) studies horizontal mergers using C\&K as a pre-merger model. Federgruen and Hu (2017b) study vertical mergers using a price competition model. ${ }^{8}$ Zhang et al. (2017) study conflict-mineral supply chains with a continuum of suppliers and retailers.

Our contribution to the supply-chain-competition literature is twofold. First, to the best of our knowledge, this is the first paper that develops a competition model for supply chains based on the market-game mechanism. This mechanism enables us to capture suppliers' seller power as well as retailers' buyer power instead of assuming that retailers are price takers in the wholesale market as in $\mathrm{C} \& \mathrm{~K}$ and A\&D. Second, as a novel research question, we study supply chain integration which

${ }^{5}$ Other than these papers, Majumder and Srinivasan (2008) study the network structure in a supply chain with potentially multiple tiers, and firms at each tier produce complementary products. Their model captures the impact of competition between two supply chain networks and leadership within a supply chain network allowing either seller power or buyer power (but not both); whereas our model captures the impact of competition among firms within a supply chain, and captures both buyer power and seller power.

${ }^{6}$ Different than our paper, C\&K consider multi-tier supply chains and vertical integration.

${ }^{7}$ We suppress product and retailer differentiation and retailer risk aversion to focus on the impact of market power. Specifically, different than A\&D, our model captures retailers' buyer power in addition to suppliers' seller power.

${ }^{8}$ Federgruen and $\mathrm{Hu}$ (2017a) provide a general framework for multi-tier price-competition models (as compared to quantity-competition models in this paper) for supply chains with multiple firms competing at each tier. Although their model captures forces of competition such as new entrants and seller power, it does not capture buyer power. 
leads to a simultaneous increase in the number of suppliers and retailers. Thus, we contribute to this literature by examining new research questions as well as by introducing a new modeling approach. Our research questions cannot be answered by projecting the existing results of the prior literature. This is evident from different insights we generate. For instance, without incorporating retailers' buyer power, the Cournot model of $\mathrm{C} \& \mathrm{~K}$ or $\mathrm{A} \& \mathrm{D}$ yields that the wholesale price is independent of the number of retailers, although it decreases with the number of suppliers. In contrast, our model yields that the wholesale price increases with the number of retailers, while decreasing with the number of suppliers. As discussed in $§ 1$, our result is consistent with OECD (1998, 2008). Also, $\mathrm{C} \& \mathrm{~K}$ and $\mathrm{A} \& \mathrm{D}$ discourage supply chain expansion because their models suggest that supply chain efficiency is maximized by either monopolizing one tier or by having duopoly in one tier and tripoly in the other tier. ${ }^{9}$ In contrast, when incorporating retailers' buyer power, our analysis shows that expansion improves the supply chain efficiency more than what $\mathrm{C} \& \mathrm{~K}$ and $\mathrm{A} \& \mathrm{D}$ show.

As we use the market-game mechanism to model the wholesale market between suppliers and retailers, we also contribute to the market-game literature. This literature usually analyzes trade among agents without incorporating production decisions (e.g., Peck and Shell 1991), but there is a handful of papers that model production decisions as well. Specifically, Dubey and Shubik (1977) extend the market-game model by incorporating production, and show that an equilibrium for this model exists under convex technology functions; Duffy and Puzzello (2014) empirically show that the presence of money improves efficiency as it acts as a coordination device; Chen et al. (2017) find a positive correlation between a firm's market share and its profit margin. The closest study to ours is by Spear (2003) who analyzes the effect of producer competition in electricity markets where producers (directly) sell electricity to end consumers. Spear (2003)'s only relevant result to our study is that the price of electricity decreases with the number of producers, and this is consistent with our result that the wholesale price decreases with the number of suppliers. Spear (2003) does not capture buyer power nor does it analyze the impact of a change in the number of buyers on prices, quantities, and profits. We contribute to the market-game literature by adapting this mechanism to supply chains and by studying supply chain expansion and integration.

\section{Model}

We study a supply chain where multiple suppliers sell a product to multiple retailers through a wholesale market. Each supplier $s \in\{1,2, \ldots, S\}$ charges a wholesale price $w$ and incurs a cost $c$

${ }^{9}$ This result holds in A\&D under no retailer differentiation. On the other hand, under perfect retailer differentiation in $A \& D$, it can be inferred (although not shown by A\&D) that supply chain expansion to include more retailers has no impact on the supply chain efficiency. In contrast, using our market-game model, we show that supply chain expansion to include more retailers always increases the supply chain efficiency. 
for each unit supplied. Let $o_{s}$ denote the quantity that supplier $s$ sells to the wholesale market (hereafter, production quantity). Then, each supplier $s$ 's profit $\pi_{s}=w o_{s}-c o_{s}$.

Each retailer $r \in\{1,2, \ldots, R\}$ procures an order quantity $q_{r}$ from the wholesale market and sells at a unit price $p_{r}$ in the retail market. We study supply chain expansion to include more retailers in different markets, so we consider retailers with independent demand. To facilitate comparison with $\mathrm{C} \& \mathrm{~K}$, we assume that each retailer has a linear inverse demand function. Specifically, $p_{r}=A-d q_{r}$, where $A>c>0$ and $d>0$, and each retailer $r$ 's profit $\pi_{r}=\left(A-d q_{r}-w\right) q_{r}$. Our main results are robust to other demand functions commonly used in the literature such as power and exponential models. In §EC.1.4 of Online Appendix, we consider retailers engaging in Cournot competition in the retail market (as in C\&K), and in $\S$ EC.1.5 of Online Appendix, we consider retailers facing demand uncertainty and supply-demand mismatches.

To model how the wholesale price is determined in the wholesale market, we adapt the models of Cournot (Cournot 1838) and the market game (Shapley and Shubik 1977) to our supply chain setting. We first present the Cournot model as a benchmark, and then present our market game.

Cournot model in the wholesale market. As a benchmark of our market-game model, we first consider the Cournot model in a two-stage Stackelberg game. This model corresponds to the two-tier supply chain model of C\&K, except that retailers in our model have independent demand; and to the A\&D model of perfect retailer differentiation. In the first stage of this Stackelberg game, each supplier determines his production quantity by considering his impact on the wholesale price and anticipating order quantities of retailers. Then, at the end of the first stage, the wholesale price is determined from an "anticipated" market-clearing condition that equates the total production quantity of suppliers with the anticipated total order quantity of retailers. In the second stage, each retailer determines her order quantity by taking the wholesale price as given. At the end of the second stage, the market clears, and each retailer receives her order quantity. In the Nash equilibrium of this game, where no supplier has an incentive to change his production quantity and no retailer has an incentive to change her order quantity, each retailer's actual order quantity in the second stage coincides with the order quantity suppliers anticipated in the first stage.

To solve for the Nash equilibrium, we derive best-response functions of suppliers and retailers by solving their optimization problems using backward induction. Throughout the paper, we use ^ to denote equilibrium and ${ }^{`}$ to denote best-response functions, and use superscript $C$ to indicate the Cournot model. For example, $\widehat{q}_{r}^{C}$ denotes retailer $r$ 's equilibrium order quantity under the Cournot model. In the second stage of the Cournot model, given a wholesale price $w$, each retailer $r$ chooses her order quantity $q_{r}$ to maximize her profit:

$$
\max _{q_{r}}\left(A-d q_{r}-w\right) q_{r}
$$


By solving (1), each retailer $r$ obtains a best-response order quantity $\breve{q}_{r}^{C}$. In the first stage of the Cournot model, each supplier $s$ chooses his production quantity $o_{s}$ by anticipating a market-clearing wholesale price $w$ to maximize his profit:

$$
\max _{w, o_{s}} w o_{s}-c o_{s} \text { s.t. } O_{-s}+o_{s}=\sum_{r=1}^{R} \breve{q}_{r}^{C} .
$$

The optimization problem in (2), where $\breve{q}_{r}^{C}$ is a function of $w$ and derived from (1), is adapted from equations (3) and (4) in $\mathrm{A} \& \mathrm{D}$. The constraint in (2) ensures that the wholesale price $w$ equates the total production quantity of suppliers with the anticipated (best-response) total order quantity of retailers. A\&D explain this condition as follows: "In the mathematical formulation of the problem, both the supply quantity $y_{i}$ [production quantity $o_{s}$ in our paper] and the wholesale market price $v[w$ in our paper $]$ appear as optimization variables, but note that only the supply quantity $y_{i}$ is a free decision of the $i$-th manufacturer [ $s$-th supplier in our paper]. The wholesale market price is implicitly determined by the wholesale-market-clearing constraint in equation (4) [the constraint in (2) in our paper], as a function of the supply quantities $y_{k}\left[o_{s}\right.$ in our paper]. Moreover, the exact same wholesale market clearing condition (4) is imposed on the decision problems of all manufacturers, and hence they all face the same wholesale-market price."

As suppliers take the wholesale price $w$ as an endogenous variable in (2), suppliers are able to influence the wholesale price, so they possess seller power. Yet, as retailers take the wholesale price $w$ as an exogenous variable in (1), retailers are price takers, so they do not possess buyer power.

Market game in the wholesale market. In our market game, the wholesale price $w$ is determined based on both suppliers' production decisions and retailers' purchasing decisions. Each supplier $s$ decides on his production quantity $o_{s}$, and we denote the total production quantity by $O=\sum_{s=1}^{S} o_{s}$. Each retailer $r$ decides on her "procurement budget" $b_{r}$ which is the payment she makes to procure a certain order quantity $q_{r} \cdot{ }^{10}$ Each retailer $r$ determines her procurement budget $b_{r}$ "without definite knowledge of what the per-unit [wholesale] price will be. At an equilibrium, this will not matter, as [wholesale] prices will be what the traders [i.e., suppliers and retailers] expect them to be" (Shapley and Shubik 1977, page 947). ${ }^{11}$ We denote the total procurement budget by $B=\sum_{r=1}^{R} b_{r}$ and the total order quantity by $Q=\sum_{r=1}^{R} q_{r}$.

\footnotetext{
${ }^{10}$ Here, the procurement budget $b_{r}$ is not a predetermined exogenous budget. The procurement budget is endogenously determined in equilibrium based on interactions between suppliers and retailers.

${ }^{11}$ Our interviews with several mid-sized retail chains have revealed that a retailer decides on a budget for the procurement of each product in most product categories, for example, tomatoes, nectarines, milk, cheese, olives, sea bass, etc., without knowing their exact prices. One of our interviewees, the owner and director of a retail chain, explains how a retailer makes procurement decisions as follows: "Before visiting a supplier, we do not determine an exact quantity to order, instead we roughly determine how much to spend on each product. We determine the exact quantity to order based on our interaction with the supplier. This reasoning applies to vegetables, fruits, cheese, olives, olive oil, milk, yogurt, etc."
} 


\begin{tabular}{|c|c|c|}
\hline $\begin{array}{l}\text { Cournot } \\
\text { model }\end{array}$ & $\begin{array}{l}\text { Each supplier } s \text { chooses his production } \\
\text { quantity } o_{s} \text { by anticipating a market- } \\
\text { clearing wholesale price } w \text {, and solves } \\
\max _{w, o_{s}} w o_{s}-c o_{s} \text { s.t. } O_{-s}+o_{s}=\sum_{r=1}^{R} \breve{q}_{r}^{C} \\
\text { At the end of stage } 1 \text {, the wholesale price } \\
w \text { is determined. }\end{array}$ & $\begin{array}{l}\text { Each retailer } r \text { chooses her order quantity } q_{r} \text { by taking } \\
\text { the wholesale price } w \text { as given, and solves } \\
\qquad \max _{q_{r}}\left(A-d q_{r}-w\right) q_{r} . \\
\text { At the end of stage } 2 \text {, each retailer } r \text { receives her } \\
\text { order quantity } q_{r} \text {. }\end{array}$ \\
\hline $\begin{array}{l}\text { Market } \\
\text { game }\end{array}$ & $\begin{array}{l}\text { Each supplier } s \text { chooses his production } \\
\text { quantity } o_{S} \text { by anticipating a market- } \\
\text { clearing wholesale price } w \text {, and solves } \\
\qquad \max _{w, O_{s}} w o_{S}-c o_{S} \text { s.t. } w=\frac{\breve{B}}{o_{s}+O_{-s}}\end{array}$ & $\begin{array}{l}\text { Each retailer } r \text { chooses a procurement budget } b_{r} \text { by } \\
\text { targeting an order quantity } q_{r} \text { and by anticipating a } \\
\text { wholesale price } w \text {, and solves } \\
\max _{b_{r}, w, q_{r}}\left(A-d q_{r}-w\right) q_{r} \text { s.t. } w=\frac{b_{r}+B_{-r}}{O}, q_{r}=\frac{b_{r} O}{b_{r}+B_{-r}} . \\
\text { The retailer's problem can be simplified as follows: } \\
\qquad \max _{w, q_{r}}\left(A-d q_{r}-w\right) q_{r} \text { s.t. } w=\frac{B_{-r}}{O-q_{r}} . \\
------ \\
\text { At the end of stage } 2 \text {, the wholesale price } w \text { is } \\
\text { determined, and each retailer } r \text { receives quantity } q_{r} \text {. }\end{array}$ \\
\hline
\end{tabular}

Figure 1 The sequence of decisions and events in the Cournot model and the market game.

The wholesale price $w$ is intuitively defined as the per-unit payment, which is the ratio of the total procurement budget (i.e., total payment) $B$ to the total production quantity $O$ :

$$
w \equiv \frac{B}{O}=\frac{\sum_{r=1}^{R} b_{r}}{\sum_{s=1}^{S} o_{s}} .
$$

Let $B_{-r}$ denote the total procurement budget of all retailers except retailer $r$. (Similarly, $O_{-s}$ denotes the total production quantity of all suppliers except supplier $s$.) Then, we can write retailer $r$ 's order quantity $q_{r}$ as follows:

$$
q_{r}=\frac{b_{r}}{w}=\frac{b_{r}}{B} O=\frac{b_{r}}{b_{r}+B_{-r}} O .
$$

Solving (4) for the procurement budget $b_{r}$, we obtain $b_{r}=\frac{q_{r} B_{-r}}{O-q_{r}}$, which is increasing in $q_{r}$, so there is a one-to-one relationship between $b_{r}$ and $q_{r}$. Because of this one-to-one relationship, a retailer's procurement budget captures her demand in the wholesale market.

We employ a two-stage Stackelberg game as in the Cournot model, while considering a simultaneous-move game in $§ E C .1 .6$ of Online Appendix, where all firms make decisions simultaneously. Figure 1 illustrates the sequence of events. In the first stage, similar to the Cournot model, each supplier determines his production quantity by considering his impact on the wholesale price. In the second stage, each retailer determines her procurement budget by targeting a certain order quantity and by considering her impact on the wholesale price. Then, at the end of the second stage, the wholesale price is determined based on these procurement budgets and production quantities according to (3), and each retailer receives her order quantity according to (4). 
To solve for the Nash equilibrium of this game, we derive best-response functions of suppliers and retailers by solving their optimization problems. In the second stage, each retailer $r$ chooses a procurement budget $b_{r}$ by anticipating a wholesale price $w$ based on (3) and by targeting an order quantity $q_{r}$ based on (4) to maximize her profit:

$$
\max _{b_{r}, w, q_{r}}\left(A-d q_{r}-w\right) q_{r} \text { s.t. (3) and (4). }
$$

Note that $b_{r}$ is retailer $r$ 's free decision variable, while $w$ and $q_{r}$ are determined subject to (3) and (4) once $b_{r}$ is determined. We can also eliminate $b_{r}$ from (5) by using its one-to-one relationship with $q_{r}$ : Substituting $b_{r}=\frac{q_{r} B_{-r}}{O-q_{r}}$ from (4) into (5) yields

$$
\max _{w, q_{r}}\left(A-d q_{r}-w\right) q_{r} \text { s.t. } w=\frac{B_{-r}}{O-q_{r}} .
$$

The endogeneity of $w$ in (6) captures retailers' buyer power as defined in OECD (2008, page 234): "just as a supplier with market power can reduce supply to force up output prices, so a buyer [retailer] with buyer power can reduce demand to force down input [wholesale] prices."

In the first stage, anticipating a market-clearing wholesale price $w$, each supplier $s$ chooses his production quantity $o_{s}$ to maximize his profit:

$$
\max _{w, o_{s}} w o_{s}-c o_{s} \text { s.t. } w=\frac{\check{B}}{o_{s}+O_{-s}} .
$$

Note that $\breve{B}=\sum_{r=1}^{R} \breve{b}_{r}$ is a function of $o_{s}$ because $\breve{b}_{r}$ is determined as a best response to $O=\sum_{s=1}^{S} o_{s}$ in (5). In (7), each supplier influences the wholesale price $w$ endogenously, similarly to the Cournot model. Because $w$ is endogenous to both suppliers and retailers, the market game captures both suppliers' seller power and retailers' buyer power. ${ }^{12}$

Before we proceed to our analysis, we summarize the main difference between the Cournot model and the market game. As illustrated in Figure 1, under the Cournot model, retailers determine order quantities at stage 2 by taking the wholesale price $w$ as exogenously given. Thus, retailers cannot cause the wholesale price to change by ordering more or less; in other words, the Cournot model does not capture retailers' buyer power. In contrast, under the market game, retailers influence $w$ endogenously at stage 2. A retailer can exercise buyer power by causing the wholesale price $w$ to change through her decision on procurement budget $b_{r}$ (or equivalently order quantity $q_{r}$ due to the one-to-one relationship between $b_{r}$ and $q_{r}$ ). This is consistent with the definition of buyer power in OECD (2008, page 9) because in the market game, a retailer "can cause the wholesale price to fall by purchasing less and cause it to rise by purchasing more." Similarly, a supplier can exercise seller power by causing the wholesale price $w$ to change through his decision on production quantity $o_{s}$. In $\S$ EC.1.2 of Online Appendix, we show that when buyer power is removed from the market game (i.e., retailers are assumed to be price takers by taking $w$ as exogenous and removing constraint

${ }^{12}$ As the market game considers competition among firms, it requires at least two retailers. It also requires the mild condition $A>\frac{R}{R-1} c$ (which holds only when $R>1$ ) to admit a non-trivial pure-strategy Nash equilibrium. 
Table 1 Summary of results in Lemma 1 and Proposition 1.

\begin{tabular}{|c|c|c|c|c|c|c|}
\hline & \multicolumn{2}{|c|}{ Equilibrium } & \multicolumn{2}{|c|}{ Larger $S$} & \multicolumn{2}{|c|}{ Larger $R$} \\
\hline & Cournot & Market game & Cournot & Market game & Cournot & Market game \\
\hline $\begin{array}{l}\text { Order } \\
\text { quantity } q\end{array}$ & $\widehat{q}^{C}=\frac{S}{S+1} \frac{A-c}{2 d} \quad>$ & $\widehat{q}=\frac{S}{S+1} \frac{A-\frac{R}{R-1} c}{2 d}$ & $\uparrow$ & $\uparrow$ & - & $\uparrow$ \\
\hline $\begin{array}{l}\text { Wholesale } \\
\text { price } w\end{array}$ & $\widehat{w}^{C}=\frac{A-c}{S+1}+c \quad>$ & $\widehat{w}=\frac{A \frac{R-1}{R}-c}{S+1}+c$ & $\downarrow$ & $\downarrow$ & - & $\uparrow$ \\
\hline
\end{tabular}

(3) from (5)), the market-game equilibrium collapses to the Cournot equilibrium. Therefore, the market game is a generalization of the Cournot model that incorporates buyer power.

\section{Supply Chain Expansion: Cournot Model vs Market Game}

In this section, we characterize and compare equilibria under the Cournot model and the market game, and analyze the impact of supply chain expansion and the resulting shifts in market power on equilibrium outcomes. In $\S 4.1$, we analyze the impact of expansion on equilibrium quantities and prices. In $\S 4.2$, we analyze the impact of expansion on profits and efficiency.

\subsection{Quantities and Prices}

We first establish the existence and uniqueness of equilibrium under the Cournot model and the market game, and then characterize the equilibrium wholesale price and order quantity. Table 1 summarizes the results of this section.

LEMMA 1. Under the Cournot model and under the market game, there exists a unique purestrategy Nash equilibrium, and the unique equilibrium is symmetric. Under the Cournot model, the equilibrium wholesale price $\widehat{w}^{C}=\frac{A-c}{S+1}+c$, and each retailer $r$ 's equilibrium order quantity $\widehat{q}_{r}^{C}=$ $\widehat{q}^{C}=\frac{S}{S+1} \frac{A-c}{2 d} \cdot{ }^{13}$ Under the market game, the equilibrium wholesale price $\widehat{w}$ and each retailer $r$ 's equilibrium order quantity $\widehat{q}_{r}=\widehat{q}$ are as follows:

$$
\widehat{w}=\frac{A \frac{R-1}{R}-c}{S+1}+c \text { and } \widehat{q}=\frac{S}{S+1} \frac{A-\frac{R}{R-1} c}{2 d} .
$$

Lemma 1 shows that the Cournot model and the market game yield similar equilibrium outcomes, although under the market game, the equilibrium wholesale price $\widehat{w}$ has an additional $\frac{R-1}{R}$ term, and the equilibrium order quantity $\widehat{q}$ has an additional $\frac{R}{R-1}$ term. Both of these terms signify the impact of the number of retailers $R$ on equilibrium outcomes under the market game. The comparison of these equilibria leads to two interesting observations. First, by incorporating the retailer's buyer power, the market game predicts a lower equilibrium wholesale price and a smaller equilibrium

${ }^{13}$ Note that A\&D's Theorem 3.11 yields the same order quantity as $\widehat{q}^{C}$ under the Cournot model when there is one product, perfect retailer differentiation, and no risk aversion. This is our only result that overlaps with A\&D. 
order quantity than the Cournot model predicts. The former result is somewhat intuitive because when retailers have buyer power, one would expect the wholesale price to be lower than the case when retailers are price takers. The latter result seems less intuitive, and its intuition is as follows. Consistent with the definition of buyer power in OECD (2008), a retailer in the market game exercises buyer power by reducing her procurement budget to reduce the wholesale price. When all retailers reduce their procurement budgets, suppliers produce less, so the equilibrium order quantity decreases. Thus, consistent with OECD (2008) (see $\S 1$ for details), the retailer's buyer power leads to a lower wholesale price and a smaller order quantity. As the number of retailers $R$ gets larger, each retailer's buyer power diminishes, and the wholesale price under the market game $\widehat{w}$ converges to that under the Cournot model $\widehat{w}^{C}$. Similarly, as $R$ gets larger, the order quantity under the market game $\widehat{q}$ converges to that under the Cournot model $\widehat{q}^{C}$.

The following proposition analyzes the impact of supply chain expansion on the equilibrium wholesale price and order quantity under the Cournot model and under the market game.

Proposition 1. (a) As the supply chain expands to include more suppliers $S$, the equilibrium wholesale price $\widehat{w}^{C}$ under the Cournot model and $\widehat{w}$ under the market game decrease, and the equilibrium order quantity $\widehat{q}^{C}$ under the Cournot model and $\widehat{q}$ under the market game increase.

(b) As the supply chain expands to include more retailers $R, \widehat{w}^{C}$ and $\widehat{q}^{C}$ under the Cournot model stay the same, whereas $\widehat{w}$ and $\widehat{q}$ under the market game increase.

Proposition 1(a) shows that under both the Cournot model and the market game, more intense competition among suppliers drives wholesale prices $\widehat{w}^{C}$ and $\widehat{w}$ down. This is intuitive as a larger number of suppliers reduces each supplier's seller power, leading to a lower wholesale price. A lower wholesale price induces each retailer to increase her order quantity, i.e., $\widehat{q}^{C}$ and $\widehat{q}$ increase.

Proposition 1(b) suggests that under the Cournot model, neither the wholesale price $\widehat{w}^{C}$ nor the order quantity $\widehat{q}^{C}$ changes with the number of retailers $R$ because retailers have no influence on the wholesale price, and hence they do not react to the increased number of retailers. ${ }^{14}$ In contrast, under the market game, the wholesale price $\widehat{w}$ increases with $R$. The intuition is as follows. As Lemma 1 shows, a retailer may reduce the wholesale price by purchasing less via reducing her procurement budget. When the number of retailers $R$ increases, each retailer's buyer power decreases, so each retailer is willing to pay more for her order due to more intense competition among retailers. Thus, each retailer increases her procurement budget $\widehat{b}$. Anticipating this increase in retailers' procurement budgets, each supplier increases his production quantity $\widehat{o}$ so as to receive a larger

\footnotetext{
${ }^{14}$ Our supplementary analysis shows that the wholesale price $\widehat{w}^{C}$ does not change with the number of retailers $R$ under any general demand function. Note that the Cournot model can mimic the result that $\widehat{w}$ increases with $R$ if an additional model feature such as a convex cost function is imposed. However, different than such models, in the market game, the result that $\widehat{w}$ increases with $R$ is driven by the retailer's buyer power.
} 
Table 2 Summary of results in Proposition 2.

\begin{tabular}{|l|c|cc|}
\hline & Larger $S$ & \multicolumn{2}{|c|}{ Larger $R$} \\
\cline { 2 - 4 } & Cournot or market game & Cournot & Market game \\
\hline Supplier profit $\pi_{s}$ & $\downarrow$ & $\uparrow$ & $\uparrow$ \\
Retailer profit $\pi_{r}$ & $\uparrow$ & No change & $\uparrow$ when $S$ is large \\
Supply chain profit $\Pi$ & $\uparrow$ & $\uparrow$ & $\uparrow$ \\
Supply chain efficiency $E$ & $\uparrow$ and concave & No change & $\uparrow$ and concave \\
\hline
\end{tabular}

share from the increased procurement budgets. The increase in $\widehat{b}$ and $\widehat{o}$ has two consequences. First, from (4), each retailer's order quantity $\widehat{q}$ increases with $R$. Second, higher production quantities from suppliers reduce $\widehat{w}$, but higher procurement budgets from retailers raise $\widehat{w}$. Proposition $1(\mathrm{~b})$ shows that the latter dominates the former, so $\widehat{w}$ increases with $R .^{15}$

Proposition 1 has important implications for the retailer's buyer power in supply chains. As we discuss in $\S 1$, the impact of buyer power on equilibrium outcomes has been identified as an important force of competition by Porter (1980) and as an important research question by C\&K. Yet, as we show in Lemma 1 and Proposition 1, the Cournot model does not capture the impact of buyer power on equilibrium outcomes $\widehat{w}^{C}$ and $\widehat{q}^{C}$. On the other hand, the market game does capture the impact of buyer power on equilibrium outcomes $\widehat{w}$ and $\widehat{q}$. Specifically, the reduced buyer power due to a larger number of retailers results in a higher wholesale price and a larger order quantity. As we discuss in $\S 1$, these results are corroborated by OECD (2008), which documents that prices and quantities decrease with buyer power. Also, OECD (2008) links buyer power to the "share of purchases in the upstream input market," so the retailer's buyer power decreases as the number of retailers increases. Thus, OECD (2008) corroborates our findings under the market game that the wholesale price and the order quantity increase with the number of retailers.

\subsection{Profits and Efficiency}

Building on our results in $\S 4.1$, we examine the supplier profit $\widehat{\pi}_{s}$, the retailer profit $\widehat{\pi}_{r}$, the supply chain profit $\widehat{\Pi}$, and its efficiency $E$ under the Cournot model and the market game. Following the literature, we define the supply chain efficiency as $E \equiv \widehat{\Pi} / \Pi^{*}$ (and $E^{C} \equiv \widehat{\Pi}^{C} / \Pi^{*}$ under the Cournot model), where $\Pi^{*}=R\left(A-d q^{*}-c\right) q^{*}$ is the centralized supply chain profit with $q^{*}=\frac{A-c}{2 d}$. Table 2 summarizes the results of this section.

The supplier profit $\widehat{\pi}_{s}$ under the market game is smaller than $\widehat{\pi}_{s}^{C}$ under the Cournot model because as we show in Lemma 1, the retailer's buyer power leads to a lower wholesale price (i.e.,

${ }^{15}$ Although the wholesale price $\widehat{w}$ and order quantity $\widehat{q}$ increase with $R$, they can move in opposite directions due to a change in another exogenous variable. For example, when the unit cost $c$ increases, $\widehat{w}$ increases and $\widehat{q}$ decreases. 
$\widehat{w}<\widehat{w}^{C}$ ) and a smaller order quantity (i.e., $\widehat{q}<\widehat{q}^{C}$ ). Yet, as Figure 2(a) shows, the retailer profit $\widehat{\pi}_{r}$ under the market game is usually larger than $\widehat{\pi}_{r}^{C}$ under the Cournot model, although $\widehat{\pi}_{r}$ can be smaller than $\widehat{\pi}_{r}^{C}$ when the number of suppliers $S$ is much larger than the number of retailers $R$. This is because increasing all retailers' buyer power has two opposing effects on each retailer $r$ 's profit. On one hand, retailer r's own buyer power increases, pushing the wholesale price down. This helps improve the retailer's profit. On the other hand, other retailers' (i.e., competitors') buyer power also increases, reducing suppliers' production and retailers' equilibrium order quantities. This has a negative impact on retailer $r$ 's profit. When the number of suppliers is large, the latter negative effect outweighs the former positive effect, so the retailer profit decreases with buyer power. Due to smaller order quantities, the supply chain profit $\widehat{\Pi}$ and efficiency $E$ under the market game are smaller than $\widehat{\Pi}^{C}$ and $E^{C}$ under the Cournot model.

We next examine how supply chain expansion affects the supplier profit $\widehat{\pi}_{s}$, the retailer profit $\widehat{\pi}_{r}$, the supply chain profit $\widehat{\Pi}$, and efficiency $E$ under the Cournot model and the market game.

Proposition 2. (a) Under the market game (resp., the Cournot model), supply chain expansion to include more suppliers $S$ reduces the supplier profit $\widehat{\pi}_{s}$ (resp., $\widehat{\pi}_{s}^{C}$ ), and raises the retailer profit $\widehat{\pi}_{r}$ (resp., $\widehat{\pi}_{r}^{C}$ ), the supply chain profit $\widehat{\Pi}$ (resp., $\widehat{\Pi}^{C}$ ), and its efficiency $E$ (resp., $E^{C}$ ).

(b) Under the Cournot model, supply chain expansion to include more retailers $R$ raises $\widehat{\pi}_{s}^{C}$ and $\widehat{\Pi}^{C}$, but has no effect on $\widehat{\pi}_{r}^{C}$ or $E^{C}$. Under the market game, supply chain expansion to include more retailers $R$ raises $\widehat{\pi}_{s}, \widehat{\Pi}$, and $E$. Also, for any $R$, there exists $\underline{S}_{R}$ such that when $S \geq \underline{S}_{R}$, $\widehat{\pi}_{r}$ under $R+1$ retailers is larger than $\widehat{\pi}_{r}$ under $R$ retailers.

(c) Under the Cournot model, the supply chain efficiency $E^{C}$ is concave in $S$. Under the market game, the supply chain efficiency $E$ is concave in both $S$ and $R$.

Proposition 2(a) shows that under both the Cournot model and the market game, having more suppliers $S$ in a supply chain reduces supplier profits $\widehat{\pi}_{s}^{C}$ and $\widehat{\pi}_{s}$, and raises retailer profits $\widehat{\pi}_{r}^{C}$ and $\widehat{\pi}_{r}$. This is intuitive because more intense competition among suppliers is expected to reduce the supplier profit, while raising the retailer profit. Yet, the increase in retailer profits outweighs the decrease in supplier profits, so supply chain profits $\widehat{\Pi}$ and $\widehat{\Pi}^{C}$ increase with $S$. Efficiency $E=\widehat{\Pi} / \Pi^{*}$ and $E^{C}$ also increase with $S$ as the centralized supply chain profit $\Pi^{*}$ does not depend on $S$.

Proposition 2(b) shows that under both the Cournot model and the market game, having more retailers $R$ in a supply chain raises supplier profits $\widehat{\pi}_{s}^{C}$ and $\widehat{\pi}_{s}$. This is mainly because having more retailers leads to more demand and hence a larger profit for each supplier. More interestingly, the impact of having more retailers $R$ on the retailer profit differs under the Cournot model and the market game. Specifically, under the Cournot model, the retailer profit $\widehat{\pi}_{r}^{C}$ does not change with $R$ because retailers have no influence on the wholesale price and hence on each other's profit. 


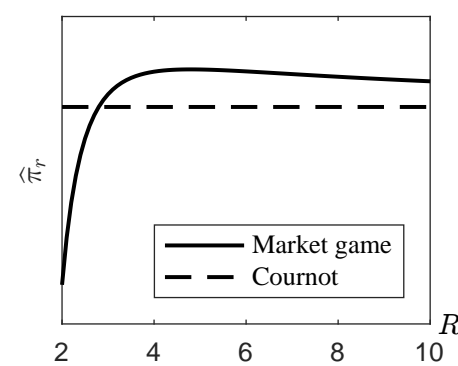

(a) Comparison of $\widehat{\pi}_{r}$ under $S=10$.

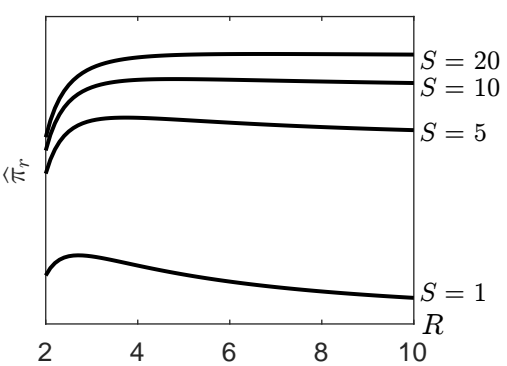

(b) $\widehat{\pi}_{r}$ as a function of $R$.

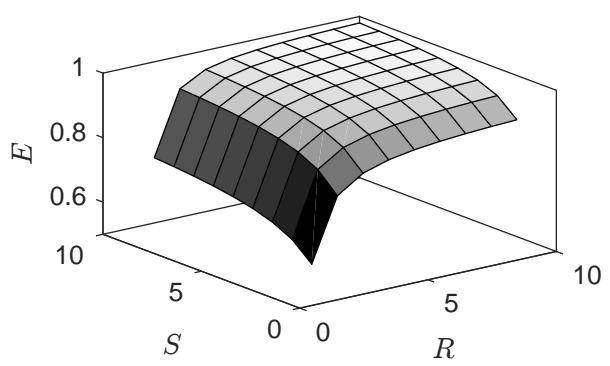

(c) $E$ as a function of $S$ and $R$.

Figure 2 (a) The comparison of the retailer profit under the Cournot model and the market game, (b) the retailer profit $\widehat{\pi}_{r}$ as a function of the number of retailers $R$ given different values of the number of suppliers $S$, and (c) the supply chain efficiency $E$ as a function of $S$ and $R$. The setting is such that the inverse demand function $p_{r}=A-d q_{r}$, where $A=3$ and $d=0.01$, and the unit cost $c=1$.

Under the market game, the retailer profit $\widehat{\pi}_{r}$ may increase or decrease with $R$, depending on the number of suppliers $S$ in the supply chain; see Figure 2(b). ${ }^{16}$ One may expect $\widehat{\pi}_{r}$ to decrease with $R$ because when the number of retailers increases, each retailer's buyer power decreases, and the wholesale price increases. Yet, when $R$ increases, as we discuss above, each retailer increases her procurement budget $\widehat{b}$. The increase in procurement budgets induces suppliers to increase their production quantities, leading to larger order quantities (Proposition 1(b)), and this improves the retailer profit $\widehat{\pi}_{r}$. When the number of suppliers is sufficiently large, Proposition 2(b) shows that the positive effect of the increase in order quantities outweighs the negative effect of the increase in the wholesale price, and hence $\widehat{\pi}_{r}$ increases with $R$.

Proposition 2(b) further shows that the supply chain profit increases with $R$ under both models. Recall that each retailer joining the supply chain has independent demand, and hence the total demand increases with $R$. This increase in the total demand explains the increased supply chain profit $\widehat{\Pi}^{C}$ under the Cournot model. However, this result is not trivial under the market game because the retailer profit $\widehat{\pi}_{r}$ can decrease with $R$. Proposition $2(\mathrm{~b})$ establishes that even if $\widehat{\pi}_{r}$ decreases with $R$, the increase in $\widehat{\pi}_{s}$ outweighs the potential decrease in $\widehat{\pi}_{r}$, so the supply chain profit $\widehat{\Pi}$ always increases with $R$. However, the higher total demand raises $\widehat{\Pi}$ (resp., $\widehat{\Pi}^{C}$ ) and $\Pi^{*}$ proportionately (see (15) in Appendix), so the positive effect of the higher total demand is not carried over to the supply chain efficiency $E=\widehat{\Pi} / \Pi^{*}$ (resp., $E^{C}=\widehat{\Pi}^{C} / \Pi^{*}$ ). Thus, whereas $R$ has no impact on efficiency $E^{C}$ under the Cournot model, efficiency $E$ increases with $R$ under the

\footnotetext{
${ }^{16}$ Nagarajan and Bassok (2008) show that as the number of suppliers increases, the supplier profit decreases, and the retailer profit (called the assembler's profit in their paper) increases. These intuitive results are consistent with our corresponding results under the market game. Different from Nagarajan and Bassok (2008), our model further shows that the retailer profit may increase with the number of retailers if the number of suppliers is sufficiently large. Furthermore, our market game model explicitly characterizes the equilibrium order quantity and wholesale price, and captures the impact of supply chain expansion on these equilibrium values.
} 
market game as the equilibrium order quantity $\widehat{q}$ increases and converges to the centralized order quantity $q^{*}$ that maximizes the supply chain profit. Therefore, when incorporating each retailer's buyer power by using the market game, the supply chain expansion is more beneficial (in terms of efficiency) than what prior literature has shown without accounting for such buyer power. ${ }^{17}$

Finally, Proposition 2(c) shows that under the market game, the supply chain efficiency $E$ is concave in the number of suppliers $S$ and in the number of retailers $R$. Proposition 2(a), (b), and (c) together suggest that the supply chain efficiency not only increases with supply chain expansion, but it also increases quite fast when the number of suppliers and retailers are small. For example, in Figure 2(c), when the supply chain consists of two suppliers and two retailers, the supply chain efficiency $E$ is $75 \%$, and it increases to $96.6 \%$ when the supply chain consists of six suppliers and six retailers, and it increases to $98.7 \%$ when the supply chain consists of ten suppliers and ten retailers. This result suggests that encouraging supply chain expansion by reducing entry barriers (e.g., taxes on imports and exports and other transaction costs) may improve the supply chain efficiency significantly, especially in supply chains with a small number of suppliers and retailers.

Propositions 1 and 2 show that under the market game, both buyer power and seller power affect equilibrium prices, quantities, profits, and efficiency, while under the Cournot model, only seller power affects them. Thus, for the rest of the paper, we use the market game as our main model.

\section{Supply Chain Integration}

In this section, we analyze supply chain integration which leads to a simultaneous increase in the number of suppliers and retailers. When transaction costs between two local supply chains decrease sufficiently, suppliers of each supply chain start transacting with retailers of the other supply chain, leading to an integrated supply chain; see Figure 3 (we formally show this result in $\S 6)$. For example, information technologies such as standard electronic business interfaces (SEBIs) facilitate supply chain integration by reducing transaction costs between firms (Malhotra et al. 2007). Similarly, economic unions such as the European Union or trade agreements such as the EU Customs Union and North American Free Trade Agreement substantially reduce transaction costs (e.g., taxes on imports and exports) across countries, and facilitate supply chain integration.

We analyze the integration of two local supply chains that we refer to as SC-A and SC-B (see Figure 3). SC-A consists of $R_{A}(\geq 2)$ retailers and $S_{A}$ suppliers, and SC-B consists of $R_{B}(\geq 2)$ retailers and $S_{B}$ suppliers. SC-A and SC-B integrate into an aggregate supply chain that consists of

\footnotetext{
${ }^{17}$ We reach the same conclusion for the supply chain expansion to include more suppliers. In addition, this result is robust to the assumption that retailers have independent demand. In $\S$ EC.1.4, we consider the case when retailers compete for the consumer demand, and show that this insight continues to hold. Moreover, we demonstrate that ignoring retailers' buyer power by using the Cournot model can significantly underestimate the benefit of additional suppliers and retailers in the supply chain.
} 


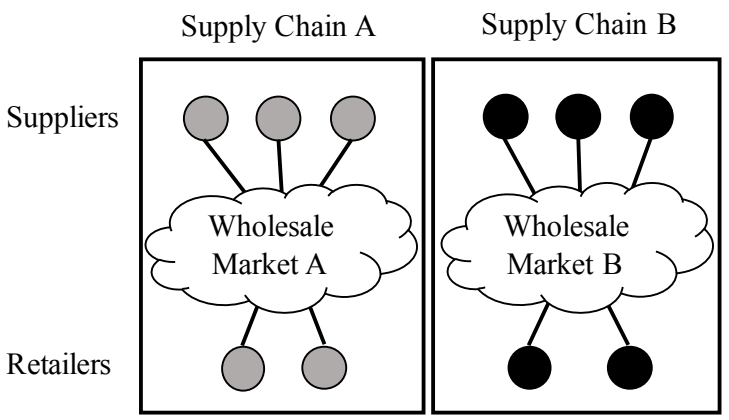

(a) Before integration

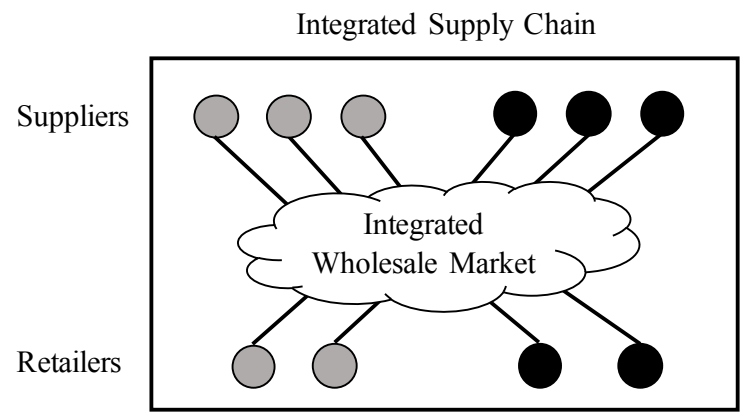

(b) After integration

Figure 3 (a) Two independent supply chains before integration and (b) the integrated supply chain.

$R\left(=R_{A}+R_{B}\right)$ retailers and $S\left(=S_{A}+S_{B}\right)$ suppliers. For example, SC-A and SC-B may represent two local supply chains that integrate after two countries sign a trade agreement.

We focus on how supply chain integration affects the total profit of firms in each local supply chain, while presenting the detailed discussion of how integration affects individual suppliers and retailers in $§$ EC.1.3 of Online Appendix. We first discuss the impact of integration on SC-A, but by symmetry, a similar intuition applies to SC-B. In Figure 4(a), the black region shows $\left(R_{A} / R, S_{A} / S\right)$ pairs where the total profit of firms in SC-A increases after integration. In the black region above the white dashed curve, integration improves both the retailer profit and the supplier profit (see Figure 4(b); note that the retail profit always improves with integration in the setting of Figure 4), and hence it improves the total profit of firms in SC-A. In the black region below the white dashed curve, integration improves the retailer profit, but reduces the supplier profit. In this region, the gain of retailers outweighs the loss of suppliers, so integration again improves the total profit of firms in SC-A. In the gray region of Figure 4(a), integration again improves the retailer profit and reduces the supplier profit; but the loss of suppliers outweighs the gain of retailers, so integration reduces the total profit of firms in SC-A. We present the precise conditions that characterize these regions in Proposition 3 below.

Proposition 3. As $S C$ - $A$ and $S C$-B integrate, the following results hold in equilibrium.

(a) The total profit of firms in $S C-m(m \in\{A, B\})$ increases if and only if

$$
R_{m} \pi(q(R, S))+\frac{A(R-1)-c R}{S+1} q(R, S)\left(\frac{S_{m}}{S}-\frac{R_{m}}{R}\right)>R_{m} \pi\left(q\left(R_{m}, S_{m}\right)\right)
$$

where $\pi(q)=(A-d q-c) q$ and $q(R, S)=\frac{S}{S+1} \frac{A-\frac{R}{R-1} c}{2 d}$.

(b) The profit of the integrated supply chain (i.e., $R \pi(q(R, S)))$ is greater than the sum of total profits of local supply chains (i.e., $\sum_{m \in\{A, B\}} R_{m} \pi\left(q\left(R_{m}, S_{m}\right)\right)$ ).

Proposition 3(a) provides a necessary and sufficient condition in (9) for each local supply chain to benefit from integration. To understand when this condition is satisfied, we discuss the expressions 


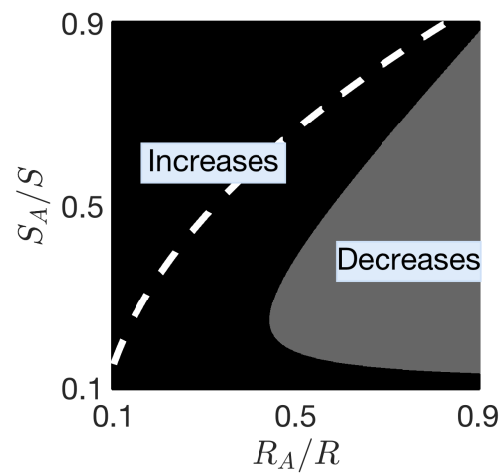

(a) A set of $\left(R_{A} / R, S_{A} / S\right)$ values where the total profit of firms in SC-A increases or decreases after integration.

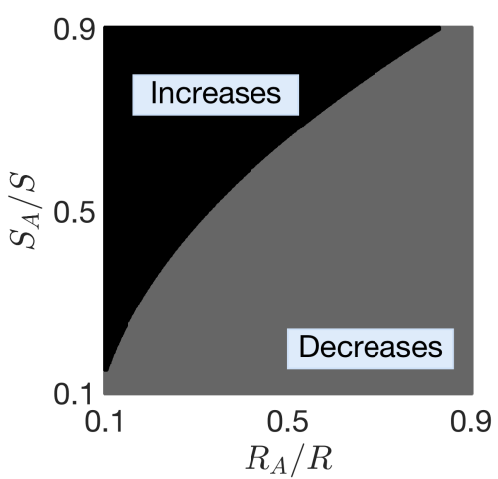

(b) A set of $\left(R_{A} / R, S_{A} / S\right)$ values where $\widehat{\pi}_{s}$ increases or decreases after integration.

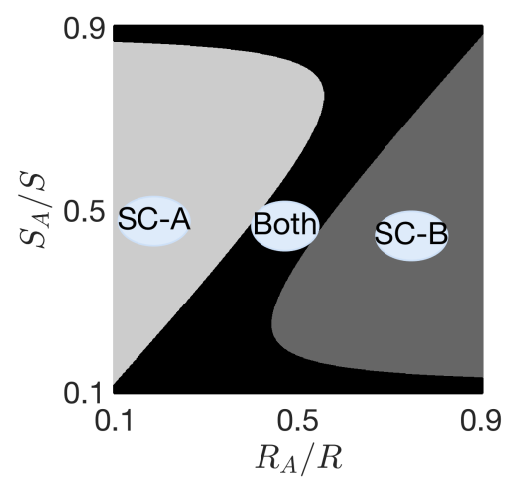

(c) A set of $\left(R_{A} / R, S_{A} / S\right)$ values where SC-A, SC-B, or both benefit from integration.

Figure 4 The comparison of the supplier profit and total profit of firms in SC-A and SC-B before and after their integration. The setting is the same as Figure 2 with $S=R=30$.

in (9). The right-hand side of (9) is the total profit of firms in SC-m $(m \in\{A, B\})$ before integration, where $\pi$ is each retailer's contribution to the supply chain profit. The left-hand side is the total profit of firms in SC-m $(m \in\{A, B\})$ after integration. Note that $R_{m} \pi(q(R, S))$ in (9) is always larger than $R_{m} \pi\left(q\left(R_{m}, S_{m}\right)\right)$ because $\pi(q)=(A-d q-c) q$ is increasing in $q$ for all $q<\frac{A-c}{2 d}$ and $q\left(R_{m}, S_{m}\right)<q(R, S)<\frac{A-c}{2 d}$ (see Proposition 1). The expression $\left(\frac{A(R-1)-c R}{S+1} q(R, S)\left(\frac{S_{m}}{S}-\frac{R_{m}}{R}\right)\right)$ in (9) corresponds to the transfer of profits from SC-m to the other local supply chain after integration. If the magnitude of this transfer of profits is sufficiently small, both local supply chains benefit from integration. Thus, a sufficient condition for both local supply chains to benefit from integration is to have the ratio of suppliers $S_{m} / S$ close to the ratio of retailers $R_{m} / R$. For instance, when SC-A consists of two suppliers and three retailers, and SC-B consists of four suppliers and six retailers, $\left(\frac{S_{A}}{S}, \frac{R_{A}}{R}\right)=\left(\frac{2}{2+4}, \frac{3}{3+6}\right)=\left(\frac{1}{3}, \frac{1}{3}\right)$, so both local supply chains benefit from integration.

Proposition 3(a) further shows that when the ratio of retailers $R_{m} / R(m \in\{A, B\})$ is significantly larger than the ratio of suppliers $S_{m} / S$, the total profit of firms in SC-m decreases after integration; see gray regions in Figure 4(c). We explain the intuition for SC-A but by symmetry, the same intuition applies to SC-B. For a more supplier-oriented SC-A (where $\frac{S_{A}}{S}>\frac{R_{A}}{R}$ ), the dominant effect of integration is expansion of markets for its suppliers. This is because after integration, a relatively large number of retailers from SC-B join as customers of suppliers in SC-A, whereas a small number of suppliers from SC-B join as competitors. However, for a more retailer-oriented SC-A (where $\frac{S_{A}}{S}<\frac{R_{A}}{R}$ ), after integration, the market share of each supplier in SC-A decreases significantly because a large number of suppliers from SC-B join as competitors, and a small number of retailers from SC-B join as customers. In this case, although each retailer increases her order quantity after integration, this positive effect does not cover the negative effect of the decrease in market share of suppliers in SC-A, so the total profit of firms in SC-A decreases. Yet, Proposition 
3(b) shows that even when integration induces a profit loss for one local supply chain, the profit gain in the other local supply chain always outweighs this loss, so the profit of the integrated supply chain is larger than the sum of total profits of local supply chains. ${ }^{18}$ Our results indicate that from a supply-chain-profit perspective, trade agreements such as the EU Single Market increases the total pie of profits, but may cause a profit loss in some member states. With the same reasoning, disintegrating from these trade agreements (e.g., recent decision of the UK to leave the EU) may improve supply chain profits; see $\S$ EC.1.3 of Online Appendix for a detailed discussion.

\section{Endogenous Entry, Exit, and Integration}

So far, we have analyzed exogenous expansion and integration, and taken the number of suppliers and retailers as given. In this section, we analyze whether there is an equilibrium number of suppliers and retailers and whether integration occurs endogenously.

We first consider an endogenous number of suppliers and retailers by incorporating firms' entry and exit decisions. Following the literature (e.g., Tyagi 1999, C\&K, Cho 2014), we analyze an equilibrium supply chain structure (i.e., the number of suppliers and retailers in equilibrium), under which no new firm benefits from entering and no existing firm benefits from exiting the supply chain. Let $c_{f}^{S}$ and $c_{f}^{R}$ be the fixed cost of entry for each supplier and each retailer, respectively. Let $\bar{S}$ and $\bar{R}$ be the number of potential suppliers and potential retailers that can enter the supply chain. Let $\widehat{\pi}_{s}(S, R)$ and $\widehat{\pi}_{r}(S, R)$ denote the equilibrium supplier profit and retailer profit, respectively, when the supply chain consists of $S$ suppliers and $R$ retailers. We define $W=\{(S, R) \in[1, \bar{S}] \times[2, \bar{R}]$ : $\widehat{\pi}_{s}(S, R) \geq 0$ and $\left.\widehat{\pi}_{r}(S, R) \geq 0\right\}$ as the set of "viable" supply chain structures where all firms make non-negative profits. Note that an equilibrium supply chain structure should not only be viable, but also require that no new supplier or retailer benefits from entering the supply chain (i.e., $(\widehat{S}, \widehat{R}) \in W,(\widehat{S}+1, \widehat{R}) \notin W$, and $(\widehat{S}, \widehat{R}+1) \notin W) .{ }^{19}$

Proposition 4. When $W$ is non-empty, it is an upper-semi lattice bounded from above. Thus, $W$ has a maximal element $(\widehat{S}, \widehat{R})$ (i.e., $S \leq \widehat{S}$ and $R \leq \widehat{R}$ for all $(S, R) \in W$ ), which is also an equilibrium supply chain structure. $\widehat{S}$ and $\widehat{R}$ are non-increasing in $c, c_{f}^{S}$, and $c_{f}^{R}$.

Proposition 4 shows that whenever $W$ is non-empty, there exists an equilibrium supply chain structure $(\widehat{S}, \widehat{R})$ where no firm benefits from entering or exiting the supply chain. Proposition 4 also implies that when fixed costs of entry $c_{f}^{S}$ and $c_{f}^{R}$ are smaller, more suppliers and more retailers

\footnotetext{
${ }^{18}$ This result suggests an opportunity to use contracts to expand the total pie of profits first and then split the benefits among local supply chains. However, such contracts would require an agreement between many independent suppliers and retailers, so it may be difficult to achieve. Such an agreement may also cause antitrust concerns.

${ }^{19}$ This implicitly assumes that firms make their entry decisions simultaneously because a sequential entry process does not lead to an equilibrium. See C\&K and Cho (2014) for further discussions.
} 
enter the supply chain, i.e., $\widehat{S}$ and $\widehat{R}$ are larger. This is because when firms incur smaller costs of entry, they make larger profits, and a larger number of firms can make positive profits. This result, together with Proposition 2, suggests that reducing entry barriers (e.g., taxes on exports and imports) facilitates supply chain expansion, which in turn improves the supply chain efficiency.

We next discuss whether integration between two local supply chains occurs endogenously. The following corollary shows that when there are no additional costs of transacting across local supply chains, either suppliers or retailers (or both) obtain larger profits when transacting in the integrated supply chain than they do when transacting in any local supply chain.

COROllaRY 1. There exist no $R_{1}, R_{2} \geq 2$ such that both the supplier profit $\widehat{\pi}_{s}$ and the retailer profit $\widehat{\pi}_{r}$ under the integrated supply chain with $R=R_{1}+R_{2}$ retailers and $S=S_{1}+S_{2}$ suppliers are smaller than those under the local supply chain with $R_{1}$ retailers and $S_{1}$ suppliers.

Corollary 1 implies that, even if there are additional costs of transacting across local supply chains, as long as those costs are sufficiently low, local supply chains are not preserved in equilibrium (leading to integration) because either suppliers or retailers (or both) are better off in the integrated supply chain. Thus, reducing transaction costs (e.g., by forming economic unions or establishing trade agreements) facilitates supply chain integration.

\section{Discussion and Conclusion}

The Cournot model is a popular model that captures several important aspects of supply chain competition, yet it fails to capture the retailer's buyer power. To overcome this limitation, we develop a competition model based on the market-game mechanism. When buyer power is removed from the market game (i.e., when retailers are assumed to be price takers), it produces the same equilibrium as the Cournot model. Therefore, the market game inherits desirable properties of the Cournot model with the added benefit of capturing the retailer's buyer power. ${ }^{20}$

Using our market-game model, we study the impact of supply chain expansion and integration on prices, quantities, profits, and efficiency. We show that an increase in the number of retailers reduces each retailer's buyer power, and leads to a higher wholesale price and larger order quantities. Although these results are consistent with OECD (1998, 2008), they cannot be shown under the

\footnotetext{
${ }^{20}$ Indeed, it is well-known that the Cournot model captures important market characteristics and provides good equilibrium predictions (e.g., Holt 1985, Cabral 2000, Carlton and Perloff 2015). The market game also inherits some limitations of the Cournot model. For example, like the Cournot model, the market game does not explicitly state how prices are formed by having firms choose output rather than price, so it requires an auctioneer to form prices. Although there are some studies that justify the price formation of the Cournot model using capacity pre-commitment (e.g., Kreps and Scheinkman 1983, Farahat et al. 2019), it is not clear whether the result that holds in a single tier will extend to the Cournot model (including C\&K and A\&D) or the market game in a two-tier supply chain; we leave this investigation for future research.
} 
Table 3 The comparison of the Cournot model and the market game.

\begin{tabular}{|l|l|l|}
\hline \multicolumn{1}{|c|}{ Cournot model } & \multicolumn{1}{c|}{ Market game } \\
\hline Advantages & $\begin{array}{l}\text { Captures industry rivalry, entry of } \\
\text { firms, and seller power. }\end{array}$ & $\begin{array}{l}\text { Captures industry rivalry, entry of } \\
\text { firms, seller power, and buyer power. }\end{array}$ \\
\hline Disadvantages & Cannot capture buyer power. & $\begin{array}{l}\text { Cannot be used for a setting with a } \\
\text { single retailer. }\end{array}$ \\
\hline $\begin{array}{l}\text { When most } \\
\text { appropriate }\end{array}$ & $\begin{array}{l}\text { Most appropriate to model multiple } \\
\text { buyers acting as price takers in a supply } \\
\text { chain (e.g., small-sized retailers). }\end{array}$ & $\begin{array}{l}\text { Most appropriate to model multiple } \\
\text { buyers that can influence prices in a } \\
\text { supply chain (e.g., mid-sized retailers). }\end{array}$ \\
\hline
\end{tabular}

Cournot model. Our analysis further generates the following insights into expansion and integration of supply chains in which retailers have independent demands:

- We show that having more retailers in a supply chain raises the supplier profit because each retailer is willing to pay more for her order when her buyer power decreases. The increased payment from retailers induces suppliers to produce more. Thus, interestingly, having more retailers in a supply chain can increase the retailer profit, especially when the number of suppliers is large. In contrast, under the Cournot model, the number of retailers has no impact on the retailer profit. Second, we show that having more suppliers in a supply chain raises the retailer profit and reduces the supplier profit. Finally, we show that the supply chain efficiency is increasing and concave in the number of suppliers and retailers. Thus, the supply chain expansion is beneficial, especially when the supply chain consists of a small number of suppliers and retailers.

- We show that as two local supply chains integrate, the profit of the integrated supply chain is greater than the sum of total profits of local supply chains. Moreover, integration raises the total profit of firms in both local supply chains when both local supply chains are retailer-oriented (i.e., with more retailers than suppliers) or both are supplier-oriented (i.e., with more suppliers than retailers). In contrast, the supply chain integration may reduce the total profit of firms in a supply chain that is more retailer-oriented than the other supply chain.

We take the first step toward introducing the market game to the supply chain literature. As the market game is a generalization of the Cournot model with the added benefit of capturing the retailer's buyer power, we believe that the market game is a good fit for any industry where the Cournot model is suitable and retailers can influence the wholesale price in a supply chain with multiple suppliers and multiple retailers. For instance, the market game may be a particularly good fit for food industries such as fruits, vegetables, dairy products, deli products, and fish. ${ }^{21}$ In a

${ }^{21}$ Food industries being a good fit for the market game is based on our interviews with several retail chains. However, a rigorous empirical study about how retailers make their purchasing decisions is an important future research to 
general setting with multiple sellers and multiple buyers, both the market game and the Cournot model have certain advantages; see Table 3. Specifically, the market game is more appropriate for influential buyers such as mid-sized retailers, whereas the Cournot model is more appropriate for non-influential buyers such as small-sized retailers (e.g., mom and pop shops) that have negligible influence on prices.

\section{Acknowledgments}

We sincerely appreciate helpful comments from the department editor Charles Corbett, the associate editor, and three reviewers. We are also grateful for helpful comments from Kenan Arifoglu, Victor DeMiguel, Stephen Spear, and seminar participants at INFORMS Annual Meeting, POMS Annual Conference, and MSOM Conference.

\section{References}

Adida, E., N. Bakshi, V. DeMiguel. 2015. Supplier capacity and intermediary profits: Can less be more? Production and Operations Management 25(4) 630-646.

Adida, E., V. DeMiguel. 2011. Supply chain competition with multiple manufacturers and retailers. Operations Research 59(1) 156-172.

Bernstein, F., F. de Vericourt. 2008. Competition for procurement contracts with service guarantees. Operations Research 56(3) 562-575.

Bernstein, F., A. Federgruen. 2003. Pricing and replenishment strategies in a distribution system with competing retailers. Operations Research 51(3) 409-426.

Bernstein, F., A. Federgruen. 2005. Decentralized supply chains with competing retailers under demand uncertainty. Management Science 51(1) 18-29.

Bernstein, F., M. Nagarajan. 2012. Competition and Cooperative Bargaining Models in Supply Chains. Foundations and Trends in Technology, Information and Operations Management, Now. URL https: //books.google.co.uk/books?id=R4M_LgEACAAJ.

Cabral, L. M. B. 2000. Industrial Organization. the MIT Press, Cambridge, MA.

Cachon, G. P., A. G. Kok. 2010. Competing manufacturers in a retail supply chain: On contractual form and coordination. Management Science 56(3) 571-589.

Carlton, D. W., J. M. Perloff. 2015. Modern Industrial Organization. Pearson, England.

Chen, G., C. G. Korpeoglu, S. E. Spear. 2017. Price stickiness and markup variations in market games. Journal of Mathematical Economics 72 95-103.

pursue. In addition to food industries, the literature suggests that the Cournot model is a good fit for industries such as automotive, consumer appliances, electronic equipment, apparel (Corbett and Karmarkar 2001), and wheat, cement, steel, cars, computers, video games, airlines, and microchips (Cabral 2000). Depending on whether retailers can influence prices, the market game or the Cournot model can be suitable for these industries. 
Cho, S-H. 2014. Horizontal mergers in multitier decentralized supply chains. Management Science 60(2) $356-379$.

Chu, L. Y., Y. Rong, H. Zheng. 2018. Strategic benefit of request for proposal/quotation. Working paper, University of Southern California.

Collard-Wexler, A., G. Gowrisankaran, R. S. Lee. 2019. Nash-in-nash bargaining: A microfoundation for applied work. Journal of Political Economy 127(1) 163-195.

Corbett, C. J., U. S. Karmarkar. 2001. Competition and structure in serial supply chains with deterministic demand. Management Science 47(7) 966-978.

Cournot, A. A. 1838. Recherches sur les Principes Mathematiques de la Theorie des Richesses. Paris: Hachette.

Crawford, G. S., A. Yorukoglu. 2012. The welfare effects of bundling in multichannel television markets. American Economic Review 102(2) 643-685.

Dubey, P., M. Shubik. 1977. A closed economic system with production and exchange modelled as a game of strategy. Journal of Mathematical Economics 4 253-287.

Duffy, J., D. Puzzello. 2014. Gift exchange versus monetary exchange: Theory and evidence. American Economic Review 104(6) 1735-1776.

Farahat, A., W. T. Huh, H. Li. 2019. On the relationship between quantity precommitment and cournot games. Operations Research 67(1) $109-122$.

Federgruen, A., M. Hu. 2017a. Competition in multi-echelon systems. Tutorials in Operations Research. INFORMS.

Federgruen, A., M. Hu. 2017b. The price and variety effects of vertical mergers. Working paper, Columbia University, New York.

Feng, Q., L. X. Lu. 2012. The strategic perils of low cost outsourcing. Management Science 58(6) 1196-1210.

Feng, Q., L. X. Lu. 2013. Supply chain contracting under competition: Bilateral bargaining vs. stackelberg. Production and Operations Management 22(3) 661-675.

Holt, C. A. 1985. An experimental test of the consistent-conjectures hypothesis. American Economic Review 75(3) 314-325.

Kreps, D.M., J. Scheinkman. 1983. Quantity precommitment and bertrand competition yield cournot outcomes. The Bell Journal of Economics 14(2) 326 - 337.

Kuo, C-W, H-S Ahn, G. Aydin. 2013. Pricing policy in a supply chain: Negotiation or posted pricing. Production and Operations Management 22(3) 626-641.

Lee, H., S. Whang. 2002. The impact of the secondary market on the supply chain. Management Science 48(6) 719-731.

Lovejoy, W. S. 2010. Bargaining chains. Management Science 56(12) 2282-2301.

Majumder, P., A. Srinivasan. 2008. Leadership and competition in network supply chains. Management Science 54(6) 1189-1204. 
Malhotra, A., S. Gosain, O. A. El Sawy. 2007. Leveraging standard electronic business interfaces to enable adaptive supply chain partnerships. Information Systems Research 18(3) 260-279.

Nagarajan, M., Y. Bassok. 2008. A bargaining framework in supply chains: The assembly problem. Management Science 54(8) 1482-1496.

Nakkas, A., Y. Xu. 2018. The impact of valuation heterogeneity on equilibrium prices in supply chain networks. Production and Operations Management, Forthcoming.

Nash, J. F. 1950. The bargaining game. Econometrica 18(2) 155-162.

Netessine, S. 2009. Supply webs: Managing, organizing, and capitalizing on global networks of suppliers. P. R. Kleindorfer, Y. J. Wind, eds., The Network Challenge: Strategy, Profit, and Risk in an Interlinked World, Chapter 13. Prentice Hall, NJ.

Netessine, S., F. Zhang. 2005. Positive vs. negative externalities in inventory management: Implications for supply chain design. Manufacturing and Service Operations Management 7(1) 58-73.

OECD. 1998. Buying power of multiproduct retailers http://www.oecd.org/competition/abuse/2379299.pdf. Accessed on November 24, 2018.

OECD. 2008. Monopsony and buyer power http://www.oecd.org/daf/competition/44445750.pdf. Accessed on November 24, 2018.

Peck, J., K. Shell. 1991. Market uncertainty: Correlated and sunspot equilibria in imperfectly competitive economies. Review of Economic Studies 58 1011-1029.

Perakis, G., G. Roels. 2007. The price of anarchy in supply chains: Quantifying the efficiency of price-only contracts. Management Science 53(8) 1249-1268.

Porter, M. E. 1980. Competitive Strategy. The Free Press, New York.

Rubinstein, A. 1982. Perfect equilibrium in a bargaining model. Econometrica 50(1) 97-109.

Shapley, L.S., M. Shubik. 1977. Trade using one commodity as a means of payment. Journal of Political Economy 85 937-968.

Shea, J. 1993. Do supply curves slope up? Quarterly Journal of Economics 108(1) 1-32.

Spear, S. E. 2003. The electricity market game. Journal of Economic Theory 109(2) 300-323.

Tang, C. S. 2016. Making OM research more relevant: "Why?" and "how?". Manufacturing and Service Operations Management 18(2) 178-183.

Tyagi, R. K. 1999. On the effects of downstream entry. Management Science 45(1) 59-73.

Zhang, H., G. Aydin, H. S. Heese. 2017. Curbing the usage of conflict minerals: A supply network perspective. Working paper, Indiana University, Bloomington.

\section{Appendix}

Proof of Lemma 1. Here we characterize equilibria under the Cournot model and the market game, while proving the existence and symmetry of equilibrium in §EC.1.1. Under the 
Cournot model, the first-order condition of the retailer's problem in (1) yields the best response $\breve{q}_{r}^{C}=\breve{q}^{C}=(A-w) / 2 d$. By substituting $\breve{q}^{C}$ and the constraint, we can rewrite $(2)$ as $\max _{w}(w-$ c) $\left[R \frac{(A-w)}{2 d}-O_{-s}\right]$. Its first-order condition evaluated at symmetric equilibrium is

$$
\left[R \frac{\left(A-\widehat{w}^{C}\right)}{2 d}-\widehat{O}_{-s}^{C}\right]-\frac{\left(\widehat{w}^{C}-c\right) R}{2 d}=\left[\frac{1}{S} R \frac{A-\widehat{w}^{C}}{2 d}\right]-\left(\widehat{w}^{C}-c\right)\left[\frac{R}{2 d}\right]=0
$$

where the first equality follows from $\widehat{O}_{-s}^{C}=(S-1) \widehat{o}^{C}=\frac{(S-1) R}{S} \widehat{q}^{C}=\frac{(S-1) R}{S} \frac{A-\widehat{w}^{C}}{2 d}$. Solving (10) for $\widehat{w}^{C}$ gives $\widehat{w}^{C}=\frac{A-c}{S+1}+c$. Thus, $\widehat{q}_{r}^{C}=\widehat{q}^{C}=\frac{A-\widehat{w}^{C}}{2 d}=\frac{A-c-\frac{A-c}{S+1}}{2 d}=\frac{S}{S+1} \frac{A-c}{2 d}$.

Under the market game, we can rewrite the retailer's problem in (5) by substituting (3) and (4) into the objective in (5):

$$
\max _{b_{r}}\left(A-d \frac{b_{r} O}{b_{r}+B_{-r}}\right) \frac{b_{r} O}{b_{r}+B_{-r}}-b_{r} .
$$

The first-order condition of (11) after imposing symmetry yields the best-response procurement budget $\breve{b}=\left(A-2 d\left(\frac{O}{R}\right)\right) \frac{O(R-1)}{R^{2}}$. Substituting $\breve{b}$ into $(7)$ yields the supplier's problem

$$
\max _{o_{s}}\left(A-2 d\left(\frac{O}{R}\right)\right) \frac{(R-1)}{R}-c o_{s} .
$$

The first-order condition of the supplier's problem evaluated at symmetric equilibrium yields

$$
\left(A-2 d\left(\frac{\widehat{O}}{R}\right)\right) \frac{(R-1)}{R}-2 d \frac{(R-1)}{R^{2}} \widehat{o}_{s}-c=0 .
$$

Letting $\widehat{q}=\widehat{O} / R$, we obtain $\widehat{q}=\frac{S}{S+1} \frac{A-\frac{R}{R-1} c}{2 d}$. Then, the equilibrium wholesale price

$$
\widehat{w}=\frac{R \widehat{b}}{\widehat{O}}=(A-2 d \widehat{q}) \frac{(R-1)}{R}=\left(A-2 d \frac{S}{S+1} \frac{A-\frac{R}{R-1} c}{2 d}\right) \frac{(R-1)}{R}=\frac{A \frac{R-1}{R}-c}{S+1}+c .
$$

Proof of Proposition 1. The proof follows from taking derivatives of $\widehat{q}^{C}, \widehat{w}^{C}, \widehat{q}$ and $\widehat{w}$ obtained in Lemma 1 with respect to $S$ and $R$.

Proof of Proposition 2. Under the Cournot model, the supplier profit and the retailer profit, respectively, can be written as

$$
\widehat{\pi}_{s}^{C}(S, R)=\frac{\left(\widehat{w}^{C}-c\right) R \widehat{q}^{C}}{S}=\frac{R(A-c)^{2}}{2 d(S+1)^{2}} \text { and } \widehat{\pi}_{r}^{C}(S, R)=\left(A-d \widehat{q}^{C}-\widehat{w}^{C}\right) \widehat{q}^{C}=\frac{S^{2}(A-c)^{2}}{4(S+1)^{2} d} .
$$

Under the market game, the supplier profit can be written as:

$$
\widehat{\pi}_{s}(S, R)=\frac{(\widehat{w}-c) R \widehat{q}}{S}=\left(\frac{A(R-1)}{(S+1) R}-\frac{c}{S+1}\right) \frac{R}{S+1} \frac{A-\frac{R}{R-1} c}{2 d}=\left(A-\frac{R}{R-1} c\right)^{2} \frac{R-1}{2 d(S+1)^{2}} .
$$

The retailer profit can be written as:

$$
\begin{aligned}
\widehat{\pi}_{r}(S, R) & =(A-d \widehat{q}-\widehat{w}) \widehat{q}=\left(A-\frac{S}{S+1} \frac{A-\frac{R}{R-1} c}{2}-\frac{A(R-1)}{(S+1) R}-\frac{S c}{S+1}\right) \frac{S}{S+1} \frac{A-\frac{R}{R-1} c}{2 d} \\
& =\left(A \frac{S R+2}{2(S+1) R}-\frac{S c(R-2)}{2(S+1)(R-1)}\right) \frac{S}{S+1} \frac{A-\frac{R}{R-1} c}{2 d}
\end{aligned}
$$


Thus, the supply chain profit under the market game is $\widehat{\Pi}=S \widehat{\pi}_{s}(S, R)+R \widehat{\pi}_{r}(S, R)=S(\widehat{w}-c) \frac{R \widehat{q}}{S}+$ $R(A-d \widehat{q}-\widehat{w}) \widehat{q}=R(A-d \widehat{q}-c) \widehat{q}$, the supply chain profit under the Cournot model is $\widehat{\Pi}^{C}=$ $R\left(A-d \widehat{q}^{C}-c\right) \widehat{q}^{C}$ and the centralized supply chain profit is $\Pi^{*}=R\left(A-d q^{*}-c\right) q^{*}$. Then, the supply chain efficiency under the market game and the Cournot model, respectively, are

$$
E=\frac{\widehat{\Pi}}{\Pi^{*}}=\frac{R(A-d \widehat{q}-c) \widehat{q}}{R\left(A-d q^{*}-c\right) q^{*}}=\frac{4 d(A-d \widehat{q}-c) \widehat{q}}{(A-c)^{2}} \text { and } E^{C}=\frac{4 d\left(A-d \widehat{q}^{C}-c\right) \widehat{q}^{C}}{(A-c)^{2}},
$$

where the third and fourth equalities follow from $q^{*}=(A-c) / 2 d$.

(a) Under the Cournot model, we can see from (12) that the supplier profit $\widehat{\pi}_{s}^{C}(S, R)$ decreases with the number of suppliers $S$ and the retailer profit $\widehat{\pi}_{r}^{C}(S, R)$ increases with $S$. Because $(A-d q-c) q$ is increasing for all $q \leq q^{*}=(A-c) / 2 d$, and $\widehat{q}^{C}(<(A-c) / 2 d)$ is increasing in $S,\left(A-d \widehat{q}^{C}-c\right) \widehat{q}^{C}$ is increasing in $S$. Thus, both $\widehat{\Pi}^{C}$ and $E^{C}$ are increasing in $S$.

Under the market game, we can see from (13) that the supplier profit $\widehat{\pi}_{s}(S, R)$ decreases with $S$. The derivative of the retailer profit $\widehat{\pi}_{r}(S, R)$ in (14) with respect to $S$ is

$$
\frac{\partial \widehat{\pi}_{r}(R, S)}{\partial S}=(A-2 d \widehat{q}-\widehat{w}) \frac{\partial \widehat{q}}{\partial S}-\widehat{q} \frac{\partial \widehat{w}}{\partial S}=\frac{A-2 d \widehat{q}}{R} \frac{\partial \widehat{q}}{\partial S}-\widehat{q} \frac{\partial \widehat{w}}{\partial S}>0,
$$

where the second equality follows because $\widehat{w}=(A-2 d \widehat{q})(R-1) / R$ (see the proof of Lemma 1) and the last inequality follows because $\widehat{q}<A / 2 d, \partial \widehat{q} / \partial S>0$, and $\partial \widehat{w} / \partial S<0$ (see Proposition 1). Because $(A-d q-c) q$ is increasing for all $q \leq q^{*}=(A-c) / 2 d$, and $\widehat{q}(<(A-c) / 2 d)$ is increasing in $S,(A-d \widehat{q}-c) \widehat{q}$ is increasing in $S$. Thus, both $\widehat{\Pi}$ and $E$ are increasing in $S$.

(b) Under the Cournot model, we can see from (12) that the supplier profit $\widehat{\pi}_{s}^{C}(S, R)$ increases with the number of retailers $R$ and the retailer profit $\widehat{\pi}_{r}^{C}(S, R)$ does not change with $R$. Because $\widehat{q}^{C}$ does not change with $R, \widehat{\Pi}^{C}$ increases with $R$ and $E^{C}$ does not change with $R$.

Under the market game, we see from (13) that the supplier profit $\widehat{\pi}_{s}(S, R)$ increases with the number of retailers $R$. Because $(A-d q-c) q$ is increasing for all $q \leq(A-c) / 2 d$, and $\widehat{q}(<(A-c) / 2 d)$ is increasing in $R$ (see Proposition 1), both $\widehat{\Pi}$ and $E$ are increasing in $R$. Furthermore, we have $\lim _{S \rightarrow \infty} \widehat{\pi}_{r}(R, S)=\left(\frac{A}{2}-\frac{c(R-2)}{2(R-1)}\right) \frac{A-\frac{R}{R-1} c}{2 d}$, which is increasing in $R$ because

$$
\frac{\partial\left(\lim _{S \rightarrow \infty} \widehat{\pi}_{r}\right)}{\partial R}=\left(-\frac{c}{2(R-1)^{2}}\right) \frac{A-\frac{R}{R-1} c}{2 d}+\left(\frac{A}{2}-\frac{c(R-2)}{2(R-1)}\right) \frac{\frac{1}{(R-1)^{2}} c}{2 d}=\frac{c^{2}}{4 d(R-1)^{2}} \frac{2}{R-1}>0 .
$$

Since $\lim _{S \rightarrow \infty} \widehat{\pi}_{r}(R, S) \in \mathbb{R}$, we have $\lim _{S \rightarrow \infty}\left(\widehat{\pi}_{r}(R+1, S)-\widehat{\pi}_{r}(R, S)\right)=\lim _{S \rightarrow \infty} \widehat{\pi}_{r}(R+1, S)-$ $\lim _{S \rightarrow \infty} \widehat{\pi}_{r}(R, S)>0$. Thus, by definition of limit, for any $R$, there exists $\underline{S}_{R}$ such that for all $S>\underline{S}_{R},\left(\widehat{\pi}_{r}(R+1, S)-\widehat{\pi}_{r}(R, S)\right)>0$.

(c) Under the Cournot model, the derivative of efficiency $E^{C}$ with respect to $S$ is

$$
\frac{\partial E^{C}}{\partial S}=\frac{4 d\left(A-2 d \widehat{q}^{C}-c\right)}{(A-c)^{2}} \frac{\partial \widehat{q}^{C}}{\partial S}=\frac{4 d\left(A-2 d \widehat{q}^{C}-c\right)}{(A-c)^{2}} \frac{1}{(S+1)^{2}} \frac{A-c}{2 d} .
$$


Under the market game, derivatives of efficiency $E$ with respect to $S$ and $R$ are as follows:

$$
\begin{aligned}
& \frac{\partial E}{\partial S}=\frac{4 d(A-2 d \widehat{q}-c)}{(A-c)^{2}} \frac{\partial \widehat{q}}{\partial S}=\frac{4 d(A-2 d \widehat{q}-c)}{(A-c)^{2}} \frac{1}{(S+1)^{2}} \frac{A-\frac{R}{R-1} c}{2 d} \\
& \frac{\partial E}{\partial R}=\frac{4 d(A-2 d \widehat{q}-c)}{(A-c)^{2}} \frac{\partial \widehat{q}}{\partial R}=\frac{4 d(A-2 d \widehat{q}-c)}{(A-c)^{2}} \frac{S}{S+1} \frac{c}{2 d(R-1)^{2}} .
\end{aligned}
$$

We have $\partial \widehat{q}^{C} / \partial S>0, \partial \widehat{q} / \partial S>0, \partial \widehat{q} / \partial R>0, \widehat{q}<(A-c) / 2 d$, and $\widehat{q}^{C}<(A-c) / 2 d$, so $\left(A-2 d \widehat{q}^{C}-c\right)$ is positive and decreasing in $S$ and $(A-2 d \widehat{q}-c)$ is positive and decreasing in $S$ and $R$. Also, $1 /(S+1)^{2}$ is decreasing in $S$ and $c / 2 d(R-1)^{2}$ is decreasing in $R$. Thus, $\partial E^{C} / \partial S$ and $\partial E / \partial S$ are decreasing (i.e., $E^{C}$ and $E$ are concave) in $S$ and $\partial E / \partial R$ is decreasing (i.e., $E$ is concave) in $R$.

Proof of Proposition 3. Let $q(R, S)=\frac{S}{S+1} \frac{A-\frac{R}{R-1} c}{2 d}$. Lemma 1 implies that in SC-m $(m \in\{A, B\})$, the equilibrium order quantity $\widetilde{q}_{m}=q\left(R_{m}, S_{m}\right)$ before integration.

(a) For this proof, we need the supplier profit $\widetilde{\pi}_{s, m}\left(\widetilde{q}_{m}\right)$ and the retailer profit $\widetilde{\pi}_{r, m}\left(\widetilde{q}_{m}\right)$ in SC-m ( $m \in\{A, B\})$ before integration. Plugging $\widetilde{o}_{m}=R_{m} \widetilde{q}_{m} / S_{m}$ into the supplier profit in SC-m ( $m \in$ $\{A, B\})$ yields: $\widetilde{\pi}_{s, m}\left(\widetilde{q}_{m}\right)=\left(\widetilde{w}_{m}-c\right) \widetilde{o}_{m}=\left(\widetilde{w}_{m}-c\right) R_{m} \widetilde{q}_{m} / S_{m}$. The retailer profit before integration is $\widetilde{\pi}_{r, m}\left(\widetilde{q}_{m}\right)=\left(A-d \widetilde{q}_{m}-\widetilde{w}_{m}\right) \widetilde{q}_{m}$. Let $\pi(q) \equiv(A-d q-c) q$. Then, we can write the total profit of firms in SC-m $(m \in\{A, B\})$ before integration as follows:

$$
\begin{aligned}
\widetilde{\Pi}_{m}\left(\widetilde{q}_{m}\right) \equiv R_{m} \widetilde{\pi}_{r, m}\left(\widetilde{q}_{m}\right)+S_{m} \widetilde{\pi}_{s, m}\left(\widetilde{q}_{m}\right) & =R_{m}\left(A-d \widetilde{q}_{m}-\widetilde{w}_{m}\right) \widetilde{q}_{m}+\left(\widetilde{w}_{m}-c\right) R_{m} \widetilde{q}_{m} \\
& =R_{m}\left(A-d \widetilde{q}_{m}-c\right) \widetilde{q}_{m}=R_{m} \pi\left(\widetilde{q}_{m}\right)=R_{m} \pi\left(q\left(R_{m}, S_{m}\right)\right) .
\end{aligned}
$$

The total profit of firms in SC-m $(m \in\{A, B\})$ after integration is

$$
\begin{aligned}
R_{m} \widehat{\pi}_{r}(\widehat{q})+S_{m} \widehat{\pi}_{s}(\widehat{q}) & =R_{m}(A-d \widehat{q}-\widehat{w}) \widehat{q}+S_{m}(\widehat{w}-c) \frac{R \widehat{q}}{S}+R_{m} c \widehat{q}-R_{m} c \widehat{q} \\
& =R_{m} \pi(\widehat{q})-R_{m} \widehat{w} \widehat{q}+(\widehat{w}-c) \frac{S_{m} R \widehat{q}}{S}+R_{m} c \widehat{q} \\
& =R_{m} \pi(q(R, S))+\left(\frac{A(R-1)-c R}{S+1}\right) q(R, S)\left(\frac{S_{m}}{S}-\frac{R_{m}}{R}\right),
\end{aligned}
$$

where the last equality follows from $\widehat{w}=\frac{A \frac{R-1}{R}-c}{S+1}+c$ and $\widehat{q}=q(R, S)$. The total profit of firms in SC-m after integration is larger than that before integration when (22) is larger than (20).

(b) As $\widetilde{q}_{m}<\widehat{q}$ from Proposition 1 , we have $\widetilde{q}_{m}<\widehat{q}<q^{*}=(A-c) / 2 d$. As $\pi(q)$ increases with $q$ for all $q<(A-c) / 2 d$, we get $\widetilde{\Pi}_{m}\left(\widetilde{q}_{m}\right)=R_{m} \pi\left(\widetilde{q}_{m}\right)<R_{m} \pi(\widehat{q})$ for $m \in\{A, B\}$ from (20). The total profit of all firms in both SC-A and SC-B satisfies $\widetilde{\Pi}_{A}\left(\widetilde{q}_{A}\right)+\widetilde{\Pi}_{B}\left(\widetilde{q}_{B}\right)<R_{A} \pi(\widehat{q})+R_{B} \pi(\widehat{q})=R \pi(\widehat{q})=\widehat{\Pi}(\widehat{q})$.

Proof of Proposition 4. We first show that non-empty $W$ is an upper-semi lattice, and that its maximal element is an equilibrium. We then show how the equilibrium changes with costs.

Suppose that $W$ is non-empty. $W$ is an upper-semi lattice if for any $\left(S_{1}, R_{1}\right) \in W$ and $\left(S_{2}, R_{2}\right) \in$ $W$, we have $\left(\max \left\{S_{1}, S_{2}\right\}, \max \left\{R_{1}, R_{2}\right\}\right) \in W$. Suppose $\left(S_{1}, R_{1}\right) \in W$ and $\left(S_{2}, R_{2}\right) \in W$. By definition $\widehat{\pi}_{j}\left(S_{i}, R_{i}\right) \geq 0$ for $j \in\{s, r\}$ and $i \in\{1,2\}$. Then, by Proposition 2 ,

$$
\widehat{\pi}_{r}\left(\max \left\{S_{1}, S_{2}\right\}, \max \left\{R_{1}, R_{2}\right\}\right) \geq \max \left\{\widehat{\pi}_{r}\left(S_{1}, \max \left\{R_{1}, R_{2}\right\}\right), \widehat{\pi}_{r}\left(S_{2}, \max \left\{R_{1}, R_{2}\right\}\right)\right\} \geq 0,
$$




$$
\widehat{\pi}_{s}\left(\max \left\{S_{1}, S_{2}\right\}, \max \left\{R_{1}, R_{2}\right\}\right) \geq \max \left\{\widehat{\pi}_{s}\left(\max \left\{S_{1}, S_{2}\right\}, R_{1}\right), \widehat{\pi}_{s}\left(\max \left\{S_{1}, S_{2}\right\}, R_{2}\right)\right\} \geq 0 .
$$

Thus, we have $\left(\max \left\{S_{1}, S_{2}\right\}, \max \left\{R_{1}, R_{2}\right\}\right) \in W$, which means $W$ is an upper-semi lattice. As $W$ is bounded by $(\bar{S}, \bar{R})$ and $W$ is an upper-semi lattice, it has a maximal element $(\widehat{S}, \widehat{R})$ (i.e., $S \leq \widehat{S}$ and $R \leq \widehat{R}$ for all $(S, R) \in W)$. By definition, $(\widehat{S}+1, \widehat{R}) \notin W$ and $(\widehat{S}, \widehat{R}+1) \notin W$, so $(\widehat{S}, \widehat{R})$ is an equilibrium structure. Under $c_{f}^{R}>0$ and $c_{f}^{S}>0$, the retailer profit and supplier profit become

$$
\begin{aligned}
& \widehat{\pi}_{r}\left(S, R \mid c, c_{f}^{R}, c_{f}^{S}\right)=\left(A \frac{S R+2}{2(S+1) R}-\frac{S c(R-2)}{2(S+1)(R-1)}\right) \frac{S}{S+1} \frac{A-\frac{R}{R-1} c}{2 d}-c_{f}^{R}, \\
& \widehat{\pi}_{s}\left(S, R \mid c, c_{f}^{R}, c_{f}^{S}\right)=\left(A-\frac{R}{R-1} c\right)^{2} \frac{R-1}{2 d(S+1)^{2}}-c_{f}^{S} .
\end{aligned}
$$

Let $\left(\widehat{S}\left(c, c_{f}^{R}, c_{f}^{S}\right), \widehat{R}\left(c, c_{f}^{R}, c_{f}^{S}\right)\right)$ be the maximal element of $W$ under costs $c, c_{f}^{R}$, and $c_{f}^{S}$. We will show that $\widehat{S}\left(c, c_{f}^{R}, c_{f}^{S}\right)$ and $\widehat{R}\left(c, c_{f}^{R}, c_{f}^{S}\right)$ are non-decreasing when we reduce $c, c_{f}^{R}$ or $c_{f}^{S}$. Suppose we change $c, c_{f}^{R}$ and $c_{f}^{S}$ to $\widetilde{c}(\leq c), \widetilde{c}_{f}^{R}\left(\leq c_{f}^{R}\right)$ and $\widetilde{c}_{f}^{S}\left(\leq c_{f}^{S}\right)$. Because $\widehat{\pi}_{r}\left(S, R \mid c, c_{f}^{R}, c_{f}^{S}\right)$ decreases with $c_{f}^{R}$ and $c$ and $\widehat{\pi}_{s}\left(S, R \mid c, c_{f}^{R}, c_{f}^{S}\right)$ decreases with $c_{f}^{S}$ and $c$, we have $\widehat{\pi}_{j}\left(\widehat{S}\left(c, c_{f}^{R}, c_{f}^{S}\right), \widehat{R}\left(c, c_{f}^{R}, c_{f}^{S}\right) \mid \widetilde{c}, \widetilde{c}_{f}^{R}, \widetilde{c}_{f}^{S}\right) \geq$ 0 for $j \in\{s, r\}$. Then, $\left(\widehat{S}\left(c, c_{f}^{R}, c_{f}^{S}\right), \widehat{R}\left(c, c_{f}^{R}, c_{f}^{S}\right)\right) \in W$ under costs $\widetilde{c}, \widetilde{c}_{f}^{R}$, and $\widetilde{c}_{f}^{S}$. Thus, by definition of the maximal element, we have $\widehat{S}\left(c, c_{f}^{R}, c_{f}^{S}\right) \leq \widehat{S}\left(\widetilde{c}, \widetilde{c}_{f}^{R}, \widetilde{c}_{f}^{S}\right)$ and $\widehat{R}\left(c, c_{f}^{R}, c_{f}^{S}\right) \leq \widehat{R}\left(\widetilde{c}, \widetilde{c}_{f}^{R}, \widetilde{c}_{f}^{S}\right)$.

Lemma A1. Let $R_{1}, S_{1}, R$, and $S$ be such that $R>R_{1} \geq 2, S>S_{1} \geq 1$, and $\widehat{w}(S, R)<\widehat{w}\left(S_{1}, R_{1}\right)$, where $\widehat{w}(S, R)=\frac{R-1}{R} \frac{A-\frac{R}{R-1} c}{S+1}+c$. Then, $\widehat{\pi}_{r}(S, R)>\widehat{\pi}_{r}\left(S_{1}, R_{1}\right)$, where $\widehat{\pi}_{r}(S, R)$ is given in (14).

Proof. We start the proof with two properties that we use in the main proof. First, note that $\pi_{r}(q)=(A-d q-w) q$ is concave in $q$ and is increasing for any $q<q^{*}(w)=\frac{A-w}{2 d}$. Second, we have

$$
\widehat{q}(S, R)=\frac{S}{S+1} \frac{A-\frac{R}{R-1} c}{2 d}<\frac{S}{S+1} \frac{A-c}{2 d}<\frac{A-c-\frac{A \frac{R-1}{R}-c}{S+1}}{2 d}=\frac{A-\widehat{w}(S, R)}{2 d}=q^{*}(\widehat{w}(S, R)) .
$$

Thus, we have the following inequality:

$$
\begin{aligned}
\widehat{\pi}_{r}\left(S_{1}, R_{1}\right) & =\left(A-d \widehat{q}\left(S_{1}, R_{1}\right)-\widehat{w}\left(S_{1}, R_{1}\right)\right) \widehat{q}\left(S_{1}, R_{1}\right) \\
& <\left(A-d \widehat{q}\left(S_{1}, R_{1}\right)-\widehat{w}(S, R)\right) \widehat{q}\left(S_{1}, R_{1}\right)<(A-d \widehat{q}(S, R)-\widehat{w}(S, R)) \widehat{q}(S, R)=\widehat{\pi}_{r}(S, R),
\end{aligned}
$$

where the first inequality follows because $\widehat{w}(S, R)<\widehat{w}\left(S_{1}, R_{1}\right)$ and the second inequality follows because $\pi_{r}(q)$ is increasing up to $q^{*}(w)$ and $\widehat{q}\left(S_{1}, R_{1}\right)<\widehat{q}(S, R)\left(<q^{*}(\widehat{w}(S, R))\right)$ by Lemma 1 .

Proof of Corollary 1. We show that there exist no $R_{1}, S_{1}, R$, and $S$ such that $R>R_{1} \geq 2$, $S>S_{1} \geq 1, \widehat{\pi}_{s}(S, R) \leq \widehat{\pi}_{s}\left(S_{1}, R_{1}\right)$, and $\widehat{\pi}_{r}(S, R) \leq \widehat{\pi}_{r}\left(S_{1}, R_{1}\right)$. Suppose the contrary. By Lemma A1, a necessary condition for $\widehat{\pi}_{r}(S, R) \leq \widehat{\pi}_{r}\left(S_{1}, R_{1}\right)$ is that $\widehat{w}(S, R) \geq \widehat{w}\left(S_{1}, R_{1}\right)$, which requires

$$
\frac{R-1}{R} \frac{A-\frac{R}{R-1} c}{S+1} \geq \frac{R_{1}-1}{R_{1}} \frac{A-\frac{R_{1}}{R_{1}-1} c}{S_{1}+1} .
$$

In order for suppliers to have larger profits in the local supply chain, we need

$$
\widehat{\pi}_{s}(S, R)=\left(A-\frac{R}{R-1} c\right)^{2} \frac{R-1}{2 d(S+1)^{2}} \leq\left(A-\frac{R_{1}}{R_{1}-1} c\right)^{2} \frac{R_{1}-1}{2 d\left(S_{1}+1\right)^{2}}=\widehat{\pi}_{s}\left(S_{1}, R_{1}\right) .
$$


Starting with (23) and then imposing (24), we can see that

$$
\begin{aligned}
\frac{\left(R_{1}-1\right)^{2}}{R_{1}^{2}} \frac{\left(A-\frac{R_{1}}{R_{1}-1} c\right)^{2}}{\left(S_{1}+1\right)^{2}} & \leq \frac{(R-1)^{2}}{R^{2}} \frac{\left(A-\frac{R}{R-1} c\right)^{2}}{(S+1)^{2}}=\left(A-\frac{R}{R-1} c\right)^{2} \frac{R-1}{(S+1)^{2}} \frac{(R-1)}{R^{2}} \\
& \leq\left(A-\frac{R_{1}}{R_{1}-1} c\right)^{2} \frac{R_{1}-1}{\left(S_{1}+1\right)^{2}} \frac{(R-1)}{R^{2}}
\end{aligned}
$$

which is a contradiction because $\frac{R_{1}-1}{R_{1}^{2}}>\frac{R-1}{R^{2}}$ for any $R>R_{1} \geq 2$. 


\section{Online Appendix}

\section{EC.1. Additional Results}

\section{EC.1.1. Existence and Symmetry of Equilibrium}

We show the existence and symmetry of equilibrium under the market game, and the same approach can be used to show the existence and symmetry under the Cournot model.

We first prove that there exists a unique pure-strategy Nash equilibrium for the subgame of retailers, and that the unique equilibrium is symmetric. We then prove the existence and symmetry of pure-strategy Nash equilibrium for the Stackelberg game. Theorem 1.2 on page 34 of Fudenberg and Tirole (1991) ensures the existence of a pure-strategy Nash equilibrium if (i) each supplier's and retailer's action space is a nonempty, compact, and convex subset of Euclidian space, and (ii) each supplier's and retailer's profit function is continuous and quasi-concave in his/her action. We prove each condition for retailers and suppliers, respectively.

First, for the subgame of retailers, each retailer $r$ 's action is her procurement budget $b_{r} \in$ $\mathbb{R}_{+}$, so her action set is $\mathbb{R}_{+}$. The first derivative of the retailer profit given in (11) is $\frac{\partial \pi_{r}}{\partial b_{r}}=$ $\left(A-2 d\left(\frac{b_{r} O}{b_{r}+B_{-r}}\right)\right) \frac{B_{-r} O}{\left(b_{r}+B_{-r}\right)^{2}}-1$. It is never optimal for retailer $r$ to set $b_{r}$ such that $A-$ $2 d\left(\frac{b_{r} O}{b_{r}+B_{-r}}\right)<0$ because $\partial \pi_{r} / \partial b_{r}<0$. Furthermore, as $b_{r}$ approaches infinity, retailer $r$ 's revenue approaches $(A-d O) O$ but her cost approaches infinity, so there exists an upper bound $\bar{b}$ on the optimal procurement budget, where $A-2 d\left(\frac{\bar{b} O}{\bar{b}+B_{-r}}\right) \geq 0$. Without loss of optimality, the retailer action set can be restricted to $[0, \bar{b}]$, which is compact and convex. The retailer profit $\pi_{r}$ in $(11)$ is continuous in $b_{r}$. The first derivative $\partial \pi_{r} / \partial b_{r}$ is strictly decreasing in $b_{r}$ given $O>0$ and $B_{-r}>0$, because $\frac{b_{r} O}{b_{r}+B_{-r}}$ is increasing in $b_{r}$, and $\frac{B_{-r} O}{\left(b_{r}+B_{-r}\right)^{2}}$ is decreasing in $b_{r}$. Thus, $\pi_{r}$ is concave in $b_{r}$, and hence quasi-concave in $b_{r} \in[0, \bar{b}]$. Therefore, there exists a pure-strategy Nash equilibrium for the subgame of retailers, and letting $\theta_{r}$ be the Lagrange multiplier for constraint $b_{r} \geq 0$, retailers' best responses in this subgame can be characterized by retailers' Kuhn-Tucker conditions:

$$
\begin{aligned}
& \left(A-2 d\left(\frac{\check{b}_{r} O}{\check{b}_{r}+\check{B}_{-r}}\right)\right) \frac{\check{B}_{-r} O}{\left(\check{b}_{r}+\check{B}_{-r}\right)^{2}}+\check{\theta}_{r}-1=0, \text { for all } r \in\{1,2, \ldots, R\}, \\
& \check{b}_{r} \geq 0, \check{\theta}_{r} \geq 0, \check{\theta}_{r} \check{b}_{r}=0, \text { for all } r \in\{1,2, \ldots, R\} .
\end{aligned}
$$

Suppose to the contrary that retailers' best-response procurement budgets are asymmetric. Then, there must be at least two retailers (labeled as retailer 1 and 2 without loss of generality) such that $\breve{b}_{1}>\breve{b}_{2}$. Because $\breve{b}_{1}>\breve{b}_{2}$, we have $\breve{b}_{1}>0$, and hence $\breve{\theta}_{1}=0$ from (EC.2). Furthermore, as we show earlier, $\partial \pi_{r} / \partial b_{r}$ is strictly decreasing in $b_{r}$, so $\left(A-2 d\left(\frac{\breve{b}_{1} O}{\check{B}}\right)\right) \frac{\left(\breve{B}_{-} \breve{b}_{1}\right) O}{(\check{B})^{2}}-1<\left(A-2 d\left(\frac{\breve{b}_{2} O}{\check{B}}\right)\right) \frac{\left(\breve{B}-\breve{b}_{2}\right) O}{(\breve{B})^{2}}-$ $1 \leq 0$. Thus, $\breve{b}_{1}$ cannot satisfy (EC.1), which is a contradiction. Thus, the best response should be 
symmetric. Given the total production quantity $O$, the retailer's unique symmetric best-response procurement budget is

$$
\breve{b}=\left(A-2 d\left(\frac{O}{R}\right)\right) \frac{O(R-1)}{R^{2}}>0 .
$$

Second, each supplier $s$ 's action is his production quantity $o_{s} \in \mathbb{R}_{+}$, so his action set is $\mathbb{R}_{+}$. Furthermore, supplier $s$ 's revenue $w o_{s}=\frac{\breve{B} o_{s}}{o_{s}+O_{-s}} \leq \breve{B}$. Then, the benefit of $o_{s}$ to the supplier is bounded because as we discuss above, $\breve{b}_{r}$ is bounded. The cost of $o_{s}$ is unbounded, so there exists an upper bound $\bar{o}$ on the optimal production quantity. Without loss of optimality, the supplier action set can be restricted to $[0, \bar{o}]$, which is compact and convex. By incorporating the retailer's best-response procurement budget into the supplier's objective, we obtain the supplier's objective in the Stackelberg game, which is to maximize:

$$
\frac{o_{s}}{o_{s}+O_{-s}}\left(A-2 d\left(\frac{O}{R}\right)\right) \frac{O(R-1)}{R}-c o_{s}=o_{s}\left(A-2 d\left(\frac{O}{R}\right)\right) \frac{(R-1)}{R}-c o_{s} .
$$

The first derivative of the supplier's profit with respect to $o_{s}$ is

$$
\frac{\partial \pi_{s}}{\partial o_{s}}=\left(A-2 d\left(\frac{O}{R}\right)\right) \frac{(R-1)}{R}-2 d \frac{(R-1)}{R^{2}} o_{s}-c .
$$

The second derivative $\frac{\partial^{2} \pi_{s}}{\partial o_{s}^{2}}=-4 d \frac{(R-1)}{R^{2}}<0$, so the supplier's profit is concave in $o_{s}$. Therefore, letting $\gamma_{s}$ be the Lagrange multiplier for non-negativity of $o_{s}$, the following Kuhn-Tucker conditions characterize the supplier's equilibrium:

$$
\begin{aligned}
& \left(A-2 d\left(\frac{\widehat{O}}{R}\right)\right) \frac{(R-1)}{R}-2 d \frac{(R-1)}{R^{2}} \widehat{o}_{s}-c+\widehat{\gamma}_{s}=0 \text { for all } s \in\{1,2, \ldots, S\}, \\
& \widehat{o}_{s} \geq 0, \gamma_{s} \geq 0, \widehat{\gamma}_{s} \widehat{o}_{s}=0, \text { for all } s \in\{1,2, \ldots, S\} .
\end{aligned}
$$

Suppose to the contrary that suppliers' equilibrium production quantities are asymmetric. Then, there must be at least two suppliers (labeled as supplier 1 and 2 without loss of generality) such that $\widehat{o}_{1}>\widehat{o}_{2}$. Then, $0 \leq \widehat{o}_{2}<\widehat{o}_{1}$. Because $\partial \pi_{s} / \partial o_{s}$ is decreasing as we discuss above, we have $\frac{\partial \pi_{s}}{\partial o_{s}}\left(\widehat{o}_{1}\right)<\frac{\partial \pi_{s}}{\partial o_{s}}\left(\widehat{o}_{2}\right) \leq 0$. Because $\widehat{\gamma}_{1}=0$ from (EC.5), $\widehat{o}_{1}$ cannot satisfy (EC.4). This contradicts the assumption that both $\widehat{o}_{1}$ and $\widehat{o}_{2}$ are equilibrium production quantities. Therefore, the equilibrium is symmetric. Letting $\widehat{q}=\frac{\widehat{O}}{R}$, and assuming that $A>\frac{R}{R-1} c$, the unique solution to (EC.4)-(EC.5) is $\widehat{q}=\frac{S}{S+1} \frac{A-\frac{R}{R-1} c}{2 d}$. The equilibrium wholesale price satisfies

$$
\widehat{w}=\frac{R \widehat{b}}{\widehat{O}}=(A-2 d \widehat{q}) \frac{(R-1)}{R}=\left(A-2 d \frac{S}{S+1} \frac{A-\frac{R}{R-1} c}{2 d}\right) \frac{(R-1)}{R}=\frac{A \frac{R-1}{R}-c}{S+1}+c .
$$

\section{EC.1.2. Isolating the Impact of Buyer Power: Market Game vs Cournot Model}

In this section, we isolate the impact of buyer power on retailers' decisions by analyzing the case where retailers in the market game act as if they were price takers. To distinguish between a retailer 
with buyer power and a price-taking retailer in the market game, we use superscript $\mathrm{P}$ to denote the equilibrium or best-response functions of price-taking retailers.

When all retailers act as if they were price takers, the wholesale price $w$ no longer appears as a decision variable in the retailer's problem in (5). Thus, we can rewrite the retailer's problem in (5) by substituting $q_{r}=b_{r} / w$ into the objective in (5) as follows:

$$
\max _{b_{r}}\left(A-d \frac{b_{r}}{w}\right) \frac{b_{r}}{w}-b_{r} .
$$

The first-order condition of (EC.6) after imposing symmetry yields the best-response procurement budget $\breve{b}=\frac{(A-w) w}{2 d}$. This yields the best-response order quantity $\breve{q}=\frac{(A-w)}{2 d}$, which is exactly the same order quantity calculated for the Cournot model in the proof of Lemma 1 . Noting that the wholesale price $w$ should clear the market, we have the market-clearing condition $O=R \frac{(A-w)}{2 d}$, which yields $o_{s}=R \frac{(A-w)}{2 d}-O_{-s}$. Substituting $o_{s}$ into (7) yields the supplier's problem

$$
\max _{w}(w-c)\left[R \frac{(A-w)}{2 d}-O_{-s}\right] .
$$

Thus, the supplier's problem is exactly the same as that in the Cournot model as derived in the proof of Lemma 1. Therefore, the supplier's problem yields the same equilibrium as the one in the Cournot model where $\widehat{w}^{P}=\widehat{w}^{C}=\frac{A-c}{S+1}+c$ and $\widehat{q}^{P}=\widehat{q}^{C}=\frac{A-\widehat{w}^{C}}{2 d}=\frac{S}{S+1} \frac{A-c}{2 d}$.

The above analysis shows that when all retailers choose not to exercise buyer power (i.e., act as price takers), the equilibrium under the market game is the same as that under the Cournot model. We next investigate whether retailers ever choose to intentionally stay in the Cournot equilibrium by not exercising buyer power. To this end, we analyze whether any single retailer $r$ can improve her profit by unilaterally exercising buyer power when all others choose not to. When each supplier chooses production quantity $\widehat{o}^{P}=R \widehat{q}^{P} / S$ and all other retailers choose the price-taker procurement budget $\widehat{b}^{P}=\widehat{w}^{P} \widehat{q}^{P}$, retailer $r$ 's profit can be written as $\pi_{r}\left(b_{r}\right)=\left(A-d \frac{b_{r} \widehat{O}^{p}}{b_{r}+\widehat{B}_{-r}^{P}}\right) \frac{b_{r} \widehat{O}^{P}}{b_{r}+\widehat{B}_{-r}^{P}}-b_{r}$. Taking the first-derivative of $\pi_{r}\left(b_{r}\right)$ with respect to $b_{r}$, and evaluating at $b_{r}=\widehat{b}^{P}$ yields

$$
\pi_{r}^{\prime}\left(\widehat{b}^{P}\right)=\left(A-2 d \widehat{q}^{P}\right) \frac{(R-1) \widehat{q}^{P}}{R \widehat{b}^{P}}-1=\left(A-(A-c) \frac{S}{S+1}\right) \frac{R-1}{R\left(\frac{A-c}{S+1}+c\right)}-1=\frac{R-1}{R}-1<0 .
$$

Because $\pi_{r}^{\prime}\left(\widehat{b}^{P}\right)<0$ and $\pi_{r}^{\prime}\left(b_{r}\right)$ is decreasing in $b_{r}$, retailer $r$ can improve her profit by choosing a smaller procurement budget $b_{r}$ than the price-taker procurement budget $\widehat{b}^{P}$. When retailer $r$ chooses a smaller procurement budget $b_{r}$ than $\widehat{b}^{P}$, she obtains a lower wholesale price $w$ than $\widehat{w}^{P}$ as evident from (3), but she also reduces her order quantity as evident from (4). Therefore, retailers always choose to exercise buyer power and end up in the market game equilibrium in Lemma 1.

\section{EC.1.3. Additional Results about Supply Chain Integration}

In this section, we provide more details on how supply chain integration affects individual firms, and we illustrate our results using a practical example. 
We first discuss the impact of supply chain integration on the retailer profit and the supplier profit in SC-A, and by symmetry, a similar intuition applies to SC-B. Integration affects the retailer profit in two ways. First, the number of retailers increases by $R_{B}$ (from $R_{A}$ to $R$ ), and having more retailers in the supply chain increases the retailer profit when $S$ is sufficiently large as shown in Proposition 2(b) and Figure 2(a). Second, the number of suppliers increases by $S_{B}$ (from $S_{A}$ to $S$ ), and as shown in Proposition 2(a), having more suppliers in the supply chain raises the retailer profit. Thus, integration benefits retailers when $S$ is sufficiently large (see Lemma EC.A1(a) below). Similarly, integration affects the supplier profit in two ways. First, the number of suppliers increases by $S_{B}$ (from $S_{A}$ to $S$ ), and as shown in Proposition 2(a), having more suppliers in the supply chain reduces the supplier profit. Second, the number of retailers increases by $R_{B}$ (from $R_{A}$ to $R$ ), and as shown in Proposition 2(b), having more retailers in the supply chain raises the supplier profit. Thus, whether the supplier profit increases or decreases after integration depends on the relative magnitude of $R_{B}$ and $S_{B}$ as compared to $R_{A}$ and $S_{A}$. These relative magnitudes can be easily captured by the ratio of retailers $R_{A} / R$ and the ratio of suppliers $S_{A} / S$. For example, a small $R_{A} / R$ indicates that the number of retailers $R_{A}$ in SC-A is substantially smaller than the number of retailers $R_{B}$ in SC-B. When the ratio of retailers $R_{A} / R$ is sufficiently smaller than the ratio of suppliers $S_{A} / S$ in SC-A, the supplier profit increases after integration; the precise condition is given in Lemma EC.A1(b).

Lemma EC.A1. (a) There exist $\underline{S}_{A}, \underline{S}_{B} \in \mathbb{R}_{+}$such that when $S_{B}>\underline{S}_{B}$, the retailer profit in $S C$ - $A$ rises after integration; and when $S_{A}>\underline{S}_{A}$, the retailer profit in $S C$-B rises after integration.

(b) The supplier profit in $S C-m(m \in\{A, B\})$ increases after integration if and only if

$$
\frac{S_{m}+1}{S+1}>\frac{A-\frac{R_{m}}{R_{m}-1} c}{A-\frac{R}{R-1} c} \sqrt{\frac{R_{m}-1}{R-1}} .
$$

A sufficient condition for (EC.7) is $R_{m} / R$ (resp., $S_{m} / S$ ) being sufficiently small (resp., large).

We next illustrate Proposition 3 using the recent decision of the UK to leave the EU, popularly known as Brexit, as an example. Our focus is on examining the impact of integration and disintegration on competition among firms in supply chains, although integration and disintegration involve various other issues. Table EC.1 illustrates contributions of supplier- and retailer-related activities to the Gross Domestic Products (GDPs) of six EU members $(m \in\{1,2, \ldots, 6\})$ over contributions of these activities to the GDP of the EU. These ratios may be used as proxies for the ratio of suppliers $S_{m} / S$ and the ratio of retailers $R_{m} / R$ for each country $m$. For example, $28.2 \%$ of supplier-related activities of Germany in Table EC.1 means that supplier-related activities contributed 626.5 billion euros to the GDP of Germany, which is $28.2 \%$ of the contribution of supplier-related activities to the EU's GDP (which is 2.22 trillion euros). We use $28.2 \%$ as a proxy for the ratio of Germany's 
Table EC.1 Contributions of supplier-related (e.g., manufacturing) and retailer-related (e.g., retail trade) activities to GDPs of EU members over contributions of these activities to the GDP of the EU (source: EU 2016).

\begin{tabular}{|l|cccccc|}
\hline & Germany & Ireland & Netherlands & Greece & Spain & UK \\
\hline (1) Supplier-related activities & $28.2 \%$ & $1.7 \%$ & $4.8 \%$ & $1.1 \%$ & $7.3 \%$ & $10.8 \%$ \\
\hline (2) Retailer-related activities & $16.0 \%$ & $1.0 \%$ & $4.5 \%$ & $1.6 \%$ & $10.9 \%$ & $14.0 \%$ \\
\hline The ratio of (1) to (2) & 1.8 & 1.7 & 1.1 & 0.6 & 0.7 & 0.8 \\
\hline
\end{tabular}

number of suppliers $S_{A}$ to the EU's number of suppliers $S$. On the one hand, Proposition 3(a) shows that a local supply chain $m$ benefits from integration when its ratio of retailers $R_{m} / R$ is smaller than its ratio of suppliers $S_{m} / S$. This result suggests that supply chain integration through the EU is likely to benefit countries such as Germany, Ireland, and Netherlands, whose ratio of retailers is smaller than their ratio of suppliers. On the other hand, Proposition 3(a) suggests that supply chain integration can harm a local supply chain when its ratio of retailers is larger than its ratio of suppliers (e.g., the gray region in Figure 4(a)). For such countries, supply chains could benefit from disintegration. As for the UK, at the micro level, disintegration after Brexit may harm (resp., benefit) supply chains in industries where the ratio of British retailers is smaller (resp., larger) than the ratio of British suppliers. At the macro level, because the UK's ratio of retailers is larger than its ratio of suppliers, disintegration after Brexit may benefit supply chains in the UK on aggregate, although it can hurt supply chains in the rest of the EU.

\section{EC.1.4. Impact of Retailer Competition}

Our base model in $\S 3$ assumes that each retailer joining a supply chain has independent demand. This section analyzes the case where retailers engage in Cournot competition in the retail market. Specifically, as in $\mathrm{C} \& \mathrm{~K}$, we assume that the retail price is determined by a linear inverse demand function $p=A-d Q$, where $Q$ denotes the total order quantity of retailers. As in our base model in $\S 3$, we consider both the Cournot model and the market game in the wholesale market. Note that Cournot competition in both wholesale and retail markets corresponds to the C\&K model.

LEMma EC.A2. Under the market game in the wholesale market and Cournot competition in the retail market, the equilibrium total order quantity and wholesale price are as follows:

$$
\widehat{Q}=\frac{S R}{(S+1)(R+1)} \frac{A-\frac{R}{R-1} c}{d} \text { and } \widehat{w}=\frac{A}{S+1} \frac{R-1}{R}+\frac{S c}{S+1} .
$$

Under Cournot competition in both wholesale and retail markets, the equilibrium total order quantity and wholesale price are as follows (adapted from equations (8) and (12) in CEKK):

$$
\widehat{Q}^{C}=\frac{S R}{(S+1)(R+1)}\left(\frac{A-c}{d}\right) \text { and } \widehat{w}^{C}=\frac{A}{S+1}+\frac{S c}{S+1} .
$$

Lemma EC.A2 shows that in the market game, the wholesale price $\widehat{w}$ increases with the number of retailers $R$ (as retailers have buyer power), whereas under the Cournot model, $R$ has no impact 


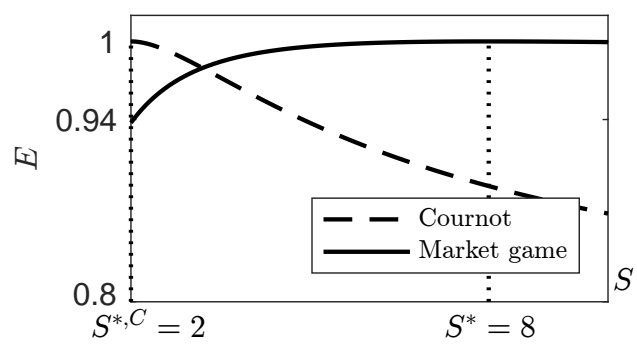

(a) $E$ as a function of $S$ when $R=3$.

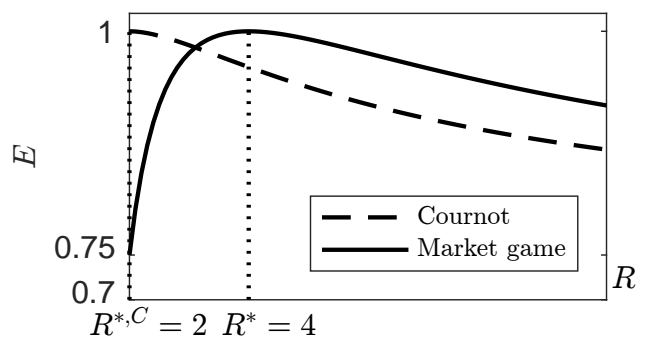

(b) $E$ as a function of $R$ when $S=3$.

Figure EC.1 The supply chain efficiency $E$ under the Cournot model and market game as a function of (a) the number of suppliers $S$ and (b) the number of retailers $R$. The setting is the same as Figure 2.

on the wholesale price $\widehat{w}^{C}$ (as retailers do not have buyer power). This finding is consistent with our finding in Proposition 1, and its intuition is as follows. In the market game, retailers consider their impact in both wholesale and retail markets, while under the Cournot model, retailers take the wholesale price given exogenously, and consider their impact only in the retail market. Thus, similar to our discussion after Proposition 1, as the number of retailers $R$ gets larger, each retailer's buyer power diminishes, and $\widehat{Q}$ and $\widehat{w}$ in (EC.8) eventually converge to $\widehat{Q}^{C}$ and $\widehat{w}^{C}$ in (EC.9).

Proposition EC.1. (a) For any number of suppliers $S$, in the market game (resp., Cournot model), the supply chain efficiency $E$ increases with the number of retailers $R$ up to an optimal number of retailers $R^{*}$ (resp., $R^{*, C}$ ), and decreases afterwards. For any $R, E$ increases with $S$ up to an optimal number of suppliers $S^{*}$ (resp., $S^{*, C}$ ), and decreases afterwards.

(b) $S^{*}>S^{*, C}$ for any $R$. Furthermore, $R^{*}=R^{*, C}=\infty$ for $S=1$, and $R^{*}>R^{*, C}$ for any $S>1$.

Proposition EC.1(a) shows that the supply chain efficiency is unimodal in the number of suppliers and retailers under both the Cournot model and the market game in the wholesale market. Note that this result is different than our result in Proposition 2. Whereas supply chain expansion to include more suppliers or more retailers always raises the supply chain efficiency in Proposition 2 (where each retailer joining a supply chain has independent demand), when retailers engage in Cournot competition, having more suppliers or retailers increases the supply chain efficiency only up to a certain level; see Figure EC.1 for illustration. The intuition for this result is essentially the same as in C\&K. Specifically, having more suppliers or retailers intensifies competition and reduces the equilibrium retail price below the desirable level for the supply chain. This is true regardless of whether the wholesale price is determined under the Cournot model or market game.

Proposition EC.1(b) shows, interestingly, that the optimal number of suppliers in the market game $S^{*}$ is larger than that under the Cournot model $S^{*, C}$. For example, Figure EC.1(a) illustrates that when the number of retailers is three, while the Cournot model prescribes $S^{*, C}=2$, the market game prescribes $S^{*}=8$. Similarly, Proposition EC.1(b) shows that (when $S>1$ ) the optimal 
number of retailers in the market game $R^{*}$ is larger than that under the Cournot model $R^{*, C}$. For example, Figure EC.1(b) demonstrates that when the number of suppliers is three, while the Cournot model prescribes $R^{*, C}=2$, the market game prescribes $R^{*}=4 .{ }^{22}$ This is because as in

$\S 4$, the retailer's total order quantity $\widehat{Q}$ under the market game is smaller than $\widehat{Q}^{C}$ under the Cournot model, and hence the retailer price decreases below the desirable level for a larger number of retailers or suppliers. In this case, ignoring retailers' buyer power by employing the Cournot model in the wholesale market leads to $25 \%$ efficiency loss. Thus, it is critical to take into account retailers' buyer power when studying supply chain competition.

\section{EC.1.5. Impact of Demand Uncertainty}

Our base model in $\S 3$ considers deterministic demand for retailers where the retail price clears the retail market. This section considers uncertain demand for retailers that may result in supplydemand mismatches in the retail market.

Each retailer $r \in\{1,2, \ldots, R\}$ faces a newsvendor problem. Before the start of a selling season, each retailer $r$ has an uncertain demand $\mathcal{D}_{r}$ and chooses an order quantity $q_{r}$ to procure from the wholesale market. We assume that $\mathcal{D}_{r}$ is independent and identically distributed with a cumulative distribution function $F$ and a density function $f$ over support $[\underline{D}, \bar{D}]$. Following the literature (e.g., Lariviere and Porteus 2001, Cachon 2004), we assume that the demand density $f$ has an increasing generalized failure rate (i.e., $\frac{f(x) x}{1-F(x)}$ is increasing in $x$ ), which is satisfied by most commonly used distributions such as normal and uniform distributions. Each retailer sells the product at a retail price $p$ and salvages any unsold unit at a salvage value $v$. As in the literature, we assume that $v<$ $c<w<p$ to avoid trivial settings. Then, each retailer $r$ 's profit $\pi_{r}=p q_{r}-(p-v) E\left[\left(q_{r}-\mathcal{D}_{r}\right)^{+}\right]-w q_{r}$.

As in $\S 3$, we consider a two-stage Stackelberg game, where suppliers make their decisions first. In the second stage of the game, each retailer $r$ chooses her procurement budget $b_{r}$ by anticipating a wholesale price $w$ and by targeting an order quantity $q_{r}$ to maximize her profit

$$
\max _{b_{r}, w, q_{r}} p q_{r}-(p-v) E\left[\left(q_{r}-\mathcal{D}_{r}\right)^{+}\right]-w q_{r} \text { s.t. (3) and (4). }
$$

The solution to (EC.10) yields retailer $r$ 's best-response order quantity $\breve{q}_{r}$ and procurement budget $\breve{b}_{r}$. In the first stage, each supplier $s$ chooses his production quantity $o_{s}$ to maximize his profit

$$
\max _{o_{s}} w o_{s}-c o_{s} \text { s.t. } w=\frac{\check{B}}{o_{s}+O_{-s}} .
$$

We first extend Lemma 1 and Proposition 1 to the case with demand uncertainty.

\footnotetext{
${ }^{22}$ Note that under the Cournot model in the wholesale market, $S^{*, C}$ and $R^{*, C}$ do not depend on parameter values $A$, $c$, or $d$. However, in the market game, $S^{*}$ and $R^{*}$ depend on the ratio $A / c$. In the case of Figure EC.1, $A / c=3$, and it leads to $R^{*}=4$ when $S=3$, and the efficiency loss under the Cournot model is $25 \%$. As another example, when $A / c=2.5, R^{*}=5$ when $S=3$, and the efficiency loss under the Cournot model increases to $44 \%$.
} 
Corollary EC.1. (a) Under both the Cournot model and the market game, there exists a unique pure-strategy Nash equilibrium, and the unique equilibrium is symmetric. Under the Cournot model, the equilibrium wholesale price $\widehat{w}^{C}$ and each retailer $r$ 's equilibrium order quantity $\widehat{q}_{r}^{C}=\widehat{q}^{C}$ satisfy $\widehat{w}^{C}=(p-v) \frac{f\left(\widehat{q}^{C}\right) \widehat{q}^{C}}{S}+c$ and $F\left(\widehat{q}^{C}\right)+\frac{f\left(\widehat{q}^{C}\right) \widehat{q}^{C}}{S}=\frac{p-c}{p-v}$. Under the market game, the equilibrium wholesale price $\widehat{w}$ and each retailer $r$ 's equilibrium order quantity $\widehat{q}_{r}=\widehat{q}$ satisfy

$$
\widehat{w}=\left((p-v) f(\widehat{q}) \widehat{q} \frac{(R-1)}{S R}\right)+c \text { and } F(\widehat{q})+\frac{f(\widehat{q}) \widehat{q}}{S}=\frac{p-\frac{R}{R-1} c}{p-v} .
$$

(b) As the supply chain expands to include more suppliers $S, \widehat{w}$ always decreases, and $\widehat{q}$ always increases. As the supply chain expands to include more retailers $R, \widehat{w}$ may increase or decrease, and $\widehat{q}$ always increases.

Corollary EC.1 characterizes the equilibrium wholesale price $\widehat{w}$ and order quantity $\widehat{q}$. Consistent with Proposition 1, the wholesale price $\widehat{w}$ in the market game depends on the number of retailers $R$, and it eventually converges to the wholesale price under the Cournot model as $R$ gets large. Yet, whereas Proposition $1(\mathrm{~b})$ in $\S 4$ shows that the wholesale price $\widehat{w}$ increases with $R$ under deterministic demand, somewhat surprisingly, Corollary EC.1 shows that depending on the demand distribution, $\widehat{w}$ may also decrease with the number of retailers $R$. Specifically, $\widehat{w}$ decreases with $R$ when demand follows a heavy-tailed Pareto distribution (e.g., an innovative product with a low probability of success but a high upside potential (e.g., Dahan and Mendelson 2001), see Figure EC.2(a)), and $\widehat{w}$ increases with $R$ when demand follows a normal distribution (e.g., a more established product with a fairly symmetric demand distribution (e.g., Gelman et al. 2003), see Figure EC.2(b)). The intuition is as follows. As in $\S 4$, having a larger number of retailers $R$ raises each retailer's procurement budget $\widehat{b}$, and each supplier increases his production quantity $\widehat{o}$ to receive a larger share from increased procurement budgets. Larger procurement budgets from retailers raise $\widehat{w}$, whereas larger production quantities from suppliers reduce $\widehat{w}$. Under a Pareto distribution, the impact of an additional order quantity on the probability of satisfying demand is small, so $\widehat{b}$ increases with $R$ more slowly than it does under a normal distribution (or under a deterministic demand as in $\S 4$ ). Thus, $\widehat{w}$ can decrease with $R$ under a Pareto distribution.

As the demand distribution affects how the wholesale price changes with the number of retailers $R$, it also affects how the retailer profit $\widehat{\pi}_{r}$ changes with $R$. Specifically, when the number of suppliers $S$ is small, $\widehat{\pi}_{r}$ increases with $R$ under a heavy-tailed Pareto distribution; see Figure EC.2(a), whereas $\widehat{\pi}_{r}$ decreases with $R$ under a normal distribution; see Figure EC.2(b). (We find that when $S$ is sufficiently large, $\widehat{\pi}_{r}$ increases with $R$ regardless of the demand distribution.) This indicates that supply chain expansion may be more beneficial to retailers in supply chains for innovative products than it is in supply chains for more established products. Nevertheless, regardless of how the retailer profit changes with $R$, supply chain expansion always improves the supply chain profit and efficiency, consistent with Proposition 2; see Corollary EC.2 below. 


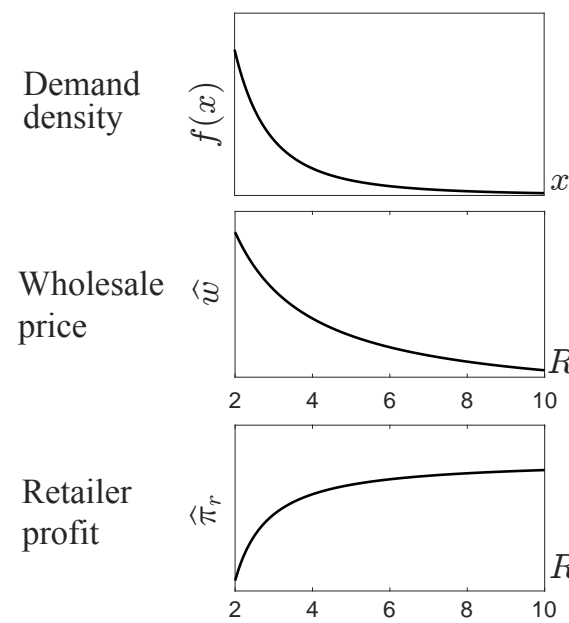

(a) $\mathcal{D}_{r} \sim$ Pareto $(125,2)$.
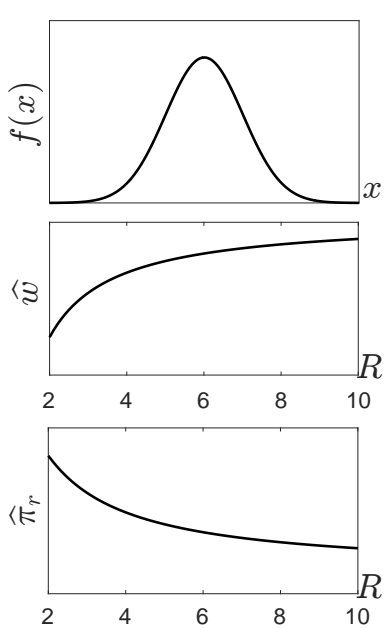

(b) $\mathcal{D}_{r} \sim \operatorname{Normal}(250,25)$.

Figure EC.2 The demand density $f$, corresponding wholesale price $\widehat{w}$, and retailer profit $\widehat{\pi}_{r}$ as the supply chain expands to include more retailers $R$. Setting: $S=2, p=\$ 115, c=\$ 35$, and $v=\$ 25$, is adopted from Chapter 17.3 of Cachon and Terwiesch (2013).

COROLlary EC.2. The supply chain profit $\widehat{\Pi}$ and its efficiency $E \equiv \widehat{\Pi} / \Pi^{*}$ increase as the supply chain expands to include more suppliers $S$ or more retailers $R$.

Finally, in Corollary EC.3 below, we obtain the same integration results as Proposition 3. Specifically, the total profit of firms in each local supply chain increases after integration when these local supply chains have similar ratio of retailers $R_{m} / R$ and ratio of suppliers $S_{m} / S$.

Corollary EC.3. As SC-A and SC-B integrate, the following results hold in equilibrium.

(a) The total profit of firms in $S C-m(m \in\{A, B\})$ increases if and only if

$$
\frac{S_{m}}{S}>\frac{R_{m}}{R}\left(1-\frac{\left(\pi(q(R, S))-\pi\left(q\left(R_{m}, S_{m}\right)\right)\right.}{(w(R, S)-c) q(R, S)}\right),
$$

where $\pi(q) \equiv(p-c) q-(p-v) E\left[\left(q-\mathcal{D}_{r}\right)^{+}\right]$, and $q(R, S)$ and $w(R, S)$ satisfy the following characterizing equations:

$$
\begin{aligned}
& F(q(R, S))+\frac{f(q(R, S)) q(R, S)}{S}=\frac{p-\frac{R}{R-1} c}{p-v}, \\
& w(R, S)=\left((p-v) f(q(R, S)) q(R, S) \frac{R-1}{S R}\right)+c .
\end{aligned}
$$

(b) The profit of the integrated supply chain is greater than the sum of profits of local supply chains.

\section{EC.1.6. Extension to a Simultaneous-Move Market Game}

In this section, we extend our main results to the case where suppliers and retailers make their decisions simultaneously under the market game. ${ }^{23}$ First, as in $\S 3$, we focus on price-setting retail-

${ }^{23}$ The discussion of the Cournot model under the simultaneous-move game is omitted because this game does not have an interior pure-strategy Nash equilibrium. 
ers under deterministic demand, and at the end of the section, we generalize our results to the newsvendor model as in $§ \mathrm{EC} .1 .5$.

The sequence of events is as follows. First, each retailer chooses her procurement budget targeting a certain order quantity, and each supplier chooses his production quantity simultaneously. The wholesale price is determined based on these procurement budgets and production quantities according to (3), and each retailer receives her order quantity according to (4). Finally, each retailer observes her demand, and then she salvages any unsold products.

We first extend Lemma 1 and Proposition 1 to the simultaneous-move market game.

COROllary EC.4. The equilibrium wholesale price $\widehat{w}$ and each retailer $r$ 's equilibrium order quantity $\widehat{q}_{r}$ under the market game are as follows:

$$
\widehat{w}=\frac{c S}{S-1} \text { and } \widehat{q}_{r}=\widehat{q}=\frac{A-\frac{c R S}{(R-1)(S-1)}}{2 d}=\frac{A-\left(\frac{R}{R-1}\right) \widehat{w}}{2 d} .
$$

The equilibrium order quantity $\widehat{q}$ increases with more suppliers $S$ or more retailers $R$. The equilibrium wholesale price $\widehat{w}$ decreases with $S$ and does not change with $R$.

Corollary EC.4 extends Proposition 1 to the simultaneous-move game. One difference between Stackelberg and simultaneous-move games is that the equilibrium wholesale price in the simultaneous-move game does not change as the supply chain expands to include more retailers $R$. The reason is as follows. With a larger $R$, each retailer increases her procurement budget $\widehat{b}$ by anticipating that the total production quantity $\widehat{O}$ is shared by more retailers. Anticipating this increase in retailers' procurement budgets, each supplier increases his production quantity $\widehat{o}$ so as to receive a larger share from the increased procurement budgets. Although higher procurement budgets from retailers increase the equilibrium wholesale price $\widehat{w}$, this increase is offset by the decrease in $\widehat{w}$ due to the higher production quantities. As a result, the equilibrium wholesale price $\widehat{w}$ does not change with the number of retailers $R$. Note that in the Stackelberg game, even though suppliers increase their production quantities, the resulting reduction in $\widehat{w}$ does not offset the increase in $\widehat{w}$ due to increased procurement budgets. This is why $\widehat{w}$ increases as the supply chain expands to include more retailers in $\S 4$.

We next extend Proposition 2 to the simultaneous-move market game.

Corollary EC.5. (a) Suppose that the supply chain expands to include more retailers. Then, the retailer profit $\widehat{\pi}_{r}$, supplier profit $\widehat{\pi}_{s}$, supply chain profit $\widehat{\Pi}$, and efficiency E increase.

(b) Suppose that the supply chain expands to include more suppliers. Then, the retailer profit $\widehat{\pi}_{r}$ always increases, and the supplier profit $\widehat{\pi}_{s}$ increases if and only if

$$
\frac{2 c R S^{2}}{(R-1)(S-1)(2 S-1)}>A
$$

The supply chain profit $\widehat{\Pi}$ and efficiency E increase. 
Corollary EC.5(a) shows that under the simultaneous-move market game, supply chain expansion to include more retailers benefits every firm, even retailers. This is because the equilibrium wholesale price $\widehat{w}$ does not change, but the equilibrium order quantity $\widehat{q}$ increases with $R$. Corollary EC.5(b) shows that as the supply chain expands to include more suppliers $S$, each retailer enjoys a higher profit. This is somewhat intuitive because when $S$ increases, the wholesale price $\widehat{w}$ decreases $(\widehat{w}=$ $\frac{c S}{S-1}$ in (EC.16)). A more interesting implication of Corollary EC.5(b) is that under a certain condition, the supplier profit $\widehat{\pi}_{s}$ increases with $S$. This is because having more suppliers reduces the wholesale price $\widehat{w}$, but at the same time raises the order quantity $\widehat{q}$. Even when $\widehat{\pi}_{s}$ decreases with $S$, as Corollary EC.5(b) shows, the supply chain profit $\widehat{\Pi}$ and efficiency $E=\widehat{\Pi} / \Pi^{*}$ increase with $S$. This is because the equilibrium order quantity $\widehat{q}$ increases with $S$ and approaches the optimal order quantity $q^{*}$ that maximizes the supply chain profit.

The following corollary extends Proposition 3 to the simultaneous-move market game.

Corollary EC.6. As $S C$-A and $S C$-B integrate, the following results hold in equilibrium.

(a) The total profit of firms in $S C-m(m \in\{A, B\})$ increases if and only if

$$
\frac{S_{m}}{S}>\frac{R_{m}}{R}\left(1-\frac{(S-1)\left(\pi(q(R, S))-\pi\left(q\left(R_{m}, S_{m}\right)\right)\right.}{c q(R, S)}\right)
$$

where $\pi(q) \equiv(A-d q-c) q$ and $q(R, S) \equiv \frac{A-\frac{c R S}{(R-1)(S-1)}}{2 d}$.

(b) The profit of the integrated supply chain is greater than the sum of total profits of local supply chains.

Corollary EC.6 shows that the impact of supply chain integration under the simultaneous-move game is qualitatively similar to that under the Stackelberg game. Both models suggest that a sufficient condition for both supply chains to benefit from integration is to have the ratio of retailers $R_{A} / R$ close to the ratio of suppliers $S_{A} / S$ (see $\S 4$ for detailed discussion).

Finally, we extend Corollaries EC.4-EC.6 to the simultaneous-move market game where retailers have uncertain demand.

COROLlary EC.7. When retailers have uncertain demand, the equilibrium wholesale price $\widehat{w}$ and each retailer $r$ 's equilibrium order quantity $\widehat{q}_{r}$ under the market game are as follows:

$$
\widehat{w}=\frac{c S}{S-1} \text { and } \widehat{q}_{r}=\widehat{q}=F^{-1}\left(\frac{p-\frac{c R S}{(R-1)(S-1)}}{p-v}\right)=F^{-1}\left(\frac{p-\left(\frac{R}{R-1}\right) \widehat{w}}{p-v}\right) .
$$

The equilibrium order quantity $\widehat{q}$ increases with more suppliers $S$ or more retailers $R$. The equilibrium wholesale price $\widehat{w}$ decreases with $S$ and does not change with $R$.

Corollary EC.8. (a) Suppose that the supply chain expands to include more retailers with uncertain demand. Then, the retailer profit $\widehat{\pi}_{r}$, supplier profit $\widehat{\pi}_{s}$, supply chain profit $\widehat{\Pi}$, and efficiency 
E increase.

(b) Suppose that the supply chain expands to include more suppliers. Then, the retailer profit $\widehat{\pi}_{r}$ always increases, and the supplier profit $\widehat{\pi}_{s}$ increases if and only if

$$
\frac{y}{(p-v)(2 S-1)}>F^{-1}\left(\frac{p-y}{p-v}\right) f\left(F^{-1}\left(\frac{p-y}{p-v}\right)\right), \text { where } y=\frac{c R S}{(R-1)(S-1)} .
$$

The supply chain profit $\widehat{\Pi}$ and efficiency E increase.

Corollary EC.9. When retailers have uncertain demand, $S C$ - $A$ and $S C$ - $B$ integrate, the following results hold in equilibrium.

(a) The total profit of firms in $S C-m(m \in\{A, B\})$ increases if and only if

$$
\frac{S_{m}}{S}>\frac{R_{m}}{R}\left(1-\frac{(S-1)\left(\pi(q(R, S))-\pi\left(q\left(R_{m}, S_{m}\right)\right)\right.}{c q(R, S)}\right),
$$

where $\pi(q) \equiv(p-c) q-(p-v) E\left[\left(q-\mathcal{D}_{r}\right)^{+}\right]$and $q(R, S) \equiv F^{-1}\left(\frac{p-\frac{c R S}{(R-1)(S-1)}}{p-v}\right)$.

(b) The profit of the integrated supply chain is greater than the sum of total profits of local supply chains.

\section{EC.2. Proofs of Additional Results}

Proof of Lemma EC.A1. (a) In SC-m $(m \in\{A, B\})$, the number of suppliers increases from $S_{m}$ to $S$ and the number of retailers increases from $R_{m}$ to $R$ after integration. Proposition 2(a) shows that the retailer profit always increases with $S$ and Proposition 2(b) shows that when $S$ is sufficiently large, the retailer profit increases with $R$. Thus, there exist $\underline{S}_{A}, \underline{S}_{B} \in \mathbb{R}_{+}$such that when $S_{m}>\underline{S}_{m}$, supply chain integration raises the retailer profit in SC-m.

(b) From (13), the supplier profit in SC-m $(m \in\{A, B\})$ increases after integration if and only if

$$
\left(A-\frac{R}{R-1} c\right)^{2} \frac{R-1}{2 d(S+1)^{2}}>\left(A-\frac{R_{m}}{R_{m}-1} c\right)^{2} \frac{R_{m}-1}{2 d\left(S_{m}+1\right)^{2}} \text {. }
$$

After simplifications, this condition leads to condition (EC.7). Because $\frac{A-\frac{R m}{R m-1} c}{A-\frac{R}{R-1} c}<1$, (EC.7) holds when $R_{m} / R$ is sufficiently small or $S_{m} / S$ is sufficiently large.

Proof of Lemma EC.A2. We first analyze the equilibrium under the market game in the wholesale market. In the second stage, given the total production quantity $O$ and other retailers' total procurement budget $B_{-r}$ and total order quantity $Q_{-r}$, each retailer $r$ determines her procurement budget $b_{r}$ by targeting an order quantity $q_{r}$ to maximize her profit

$$
\pi_{r}\left(b_{r}\right)=\left(A-d\left(q_{r}+Q_{-r}\right)\right) q_{r}-b_{r}=\left[A-d \frac{b_{r} O}{b_{r}+B_{-r}}-d Q_{-r}\right] \frac{b_{r} O}{b_{r}+B_{-r}}-b_{r} .
$$


The first-order condition of the retailer's problem after incorporating $\breve{Q}_{-r}=\frac{(R-1) O}{R}$ arising from the market-clearing condition $O=\breve{Q}$ gives the retailer's best-response $\breve{b}=\left(A-d \frac{R+1}{R} O\right) \frac{(R-1) O}{R^{2}}$. In the first stage, each supplier $s$ determines his production quantity $o_{s}$ to maximize

$$
\pi_{s}\left(o_{s}\right)=\frac{o_{s}}{o_{s}+O_{-s}} \check{B}-c o_{s}=o_{s}\left(A-d \frac{R+1}{R} o_{s}-d \frac{R+1}{R} O_{-s}\right) \frac{R-1}{R}-c o_{s} .
$$

The first-order condition of the supplier's problem above evaluated at symmetric equilibrium yields the supplier's equilibrium production quantity $\widehat{o}=\frac{R}{R+1} \frac{1}{S+1} \frac{A-\frac{R}{R-1} c}{d}$. Then, the equilibrium total order quantity $\widehat{Q}$ and wholesale price $\widehat{w}$ under the market game are

$$
\widehat{Q}=\frac{R}{R+1} \frac{S}{S+1} \frac{A-\frac{R}{R-1} c}{d} \text { and } \widehat{w}=\frac{\widehat{B}}{\widehat{O}}=\left(A-d \frac{R+1}{R} \widehat{O}\right) \frac{(R-1)}{R}=\frac{A}{S+1} \frac{R-1}{R}+\frac{S c}{S+1} .
$$

Under Cournot competition in the wholesale market, the equilibrium total order quantity $\widehat{Q}^{C}$ and wholesale price $\widehat{w}^{C}$ can be obtained from equations (8) and (12) in C\&K.

Proof of Proposition EC.1. The supply chain profit can be written as:

$$
\Pi=(A-d Q) Q-w Q+w Q-c Q=(A-c) Q-d Q^{2}=(A-c) Q-d Q^{2} .
$$

It is easy to verify that the optimal total order quantity that maximizes $\Pi$ is $Q^{*}=(A-c) / 2 d$. In this case, the supply chain efficiency $E$ under the total order quantity $Q$ can be written as:

$$
E=\frac{(A-c) Q-d Q^{2}}{(A-c) Q^{*}-d\left(Q^{*}\right)^{2}}=\frac{4 d\left[(A-c) Q-d Q^{2}\right]}{(A-c)^{2}} .
$$

Because $E$ is quadratic in $Q, E$ is increasing in $Q$ for all $Q<Q^{*}$ and decreasing in $Q$ for all $Q>Q^{*}$.

(a) We prove the result for the market game but the same logic applies to the Cournot model as well. $E$ is increasing in $S$ and $R$ as long as $\widehat{Q}<Q^{*}$ and $E$ is decreasing in $S$ and $R$ after $\widehat{Q}>Q^{*}$. Because $\widehat{Q}$ increases with $S$ and $R$ by Proposition 1, for any $S$ (resp., $R$ ), there exists $R^{*}$ (resp., $S^{*}$ ) such that $E$ increases with $R$ up to $R^{*}$ (resp., $S$ up to $S^{*}$ ) and decreases afterwards.

(b) We compare total order quantities under the Cournot model and the market game as follows:

$$
\widehat{Q}=\frac{R}{R+1} \frac{S}{S+1} \frac{A-\frac{R}{R-1} c}{d}<\frac{S}{(S+1) d} \frac{R}{R+1}(A-c)=\widehat{Q}^{C} .
$$

Take an arbitrary $R$, and suppose to the contrary that $S^{*} \leq S^{*, C}$. From the discussion above, we know that for all $S \leq S^{*, C}$, we have $\widehat{Q}^{C} \leq Q^{*}$, which means that when $S=S^{*}, C$, from (EC.23), $\widehat{Q}<\widehat{Q}^{C} \leq Q^{*}$. Then, by continuity of $\widehat{Q}$ in $S$, we can find sufficiently small $\epsilon(>0)$ such that $\widehat{Q}<Q^{*}$ for $S=S^{*, C}+\epsilon$, which means that $S^{*} \geq S^{*, C}+\epsilon>S^{*, C}$, which is a contradiction. Thus, for any $R$, we have $S^{*}>S^{*, C}$. When $S=1, \widehat{Q}^{C}<Q^{*}$ and $\widehat{Q}<Q^{*}$ for all $R$, so $R^{*}=R^{*, C}=\infty$. For any $S>1$, similar to the above discussion, when $R=R^{*, C}$, we have $\widehat{Q}<Q^{*}$, so $R^{*}>R^{*, C}$.

Proof of Corollary EC.1. (a) We show the existence and symmetry of equilibrium for the market game, but the result follows for the Cournot model as well. We first characterize the 
equilibrium under the Cournot model. Then, under the market game, we prove that there exists a unique pure-strategy Nash equilibrium for the subgame of retailers, and that the unique equilibrium is symmetric. We then prove the existence and symmetry of pure-strategy Nash equilibrium for the whole game, and characterize the equilibrium. Finally, we show comparative statics in part (b).

Cournot. Given a wholesale price $w$ in the second stage, each retailer $r$ determines her order quantity $q_{r}$ to maximize her expected profit

$$
\pi_{r}^{C}\left(q_{r}\right)=p q_{r}-(p-v) E\left[\left(q_{r}-\mathcal{D}_{r}\right)^{+}\right]-w q_{r} .
$$

By maximizing $\pi_{r}^{C}$, each retailer $r$ obtains a best-response order quantity $\breve{q}_{r}^{C}=\breve{q}^{C}=F^{-1}\left(\frac{p-w}{p-v}\right)$. Anticipating retailers' best-response order quantities, each supplier determines his production quantity and wholesale price to maximize his profit considering that the wholesale price clears the wholesale market (i.e., a market-clearing condition). Thus, each supplier $s$ solves the following problem (which is adapted from equations (3) and (4) in A\&D):

$$
\max _{o_{s}, w}(w-c) o_{s} \text { s.t. } O_{-s}+o_{s}=R F^{-1}\left(\frac{p-w}{p-v}\right) .
$$

Substituting the constraint into the objective in (EC.25), the supplier's problem can be rewritten

$$
\max _{w}(w-c)\left[R F^{-1}\left(\frac{p-w}{p-v}\right)-O_{-s}\right] .
$$

The first-order condition of the supplier's problem at symmetric equilibrium is

$$
\left[R F^{-1}\left(\frac{p-w}{p-v}\right)-\widehat{O}_{-s}^{C}\right]-\frac{R}{p-v} \frac{(w-c)}{f\left(F^{-1}\left(\frac{p-w}{p-v}\right)\right)}=\frac{R}{S} \widehat{q}^{C}-\left[\frac{R}{p-v} \frac{\left(p-F\left(\widehat{q}^{C}\right)(p-v)-c\right)}{f\left(\widehat{q}^{C}\right)}\right]=0,
$$

where the first equality follows from $\widehat{q}^{C}=F^{-1}\left(\frac{p-w}{p-v}\right)$ and $\widehat{O}^{C}=R \widehat{q}^{C}$. Solving (EC.26) for $\widehat{q}^{C}$ gives the following characterizing equation for the equilibrium order quantity $\widehat{q}^{C}$ under Cournot:

$$
F\left(\widehat{q}^{C}\right)+\frac{f\left(\widehat{q}^{C}\right) \widehat{q}^{C}}{S}=\frac{p-c}{p-v} .
$$

The equilibrium wholesale price $\widehat{w}^{C}$ under Cournot competition satisfies the following equation:

$$
\widehat{w}^{C}=p-F\left(\widehat{q}^{C}\right)(p-v)=(p-v) \frac{f\left(\widehat{q}^{C}\right) \widehat{q}^{C}}{S}+c .
$$

Market game. Step 1: Existence and Symmetry. Theorem 1.2 on page 34 of Fudenberg and Tirole (1991) ensures the existence of a pure-strategy Nash equilibrium if (i) each supplier's and retailer's action space is a nonempty, compact, and convex subset of Euclidian space, and (ii) each supplier's and retailer's profit function is continuous and quasi-concave in his/her action. We prove each condition for retailers and suppliers, respectively.

First, for the subgame of retailers, each retailer $r$ 's action is her procurement budget $b_{r} \in \mathbb{R}_{+}$, so her action set is $\mathbb{R}_{+}$. Because retailer $r$ 's order quantity $q_{r}=\frac{b_{r} O}{b_{r}+B_{-r}}$, and the retailer profit in (EC.10) diverges to negative infinity as $q_{r}$ approaches infinity (since $c>v$ ), there exists an upper 
bound $\bar{b}$ on the optimal procurement budget. Without loss of optimality, the retailer's action set can be restricted to $[0, \bar{b}]$, which is compact and convex. The retailer profit $\pi_{r}$ in (EC.10) is continuous in $b_{r}$. The first derivative of $\pi_{r}$ with respect to $b_{r}, \frac{\partial \pi_{r}}{\partial b_{r}}=\left(p-(p-v) F\left(\frac{b_{r} O}{b_{r}+B_{-r}}\right)\right) \frac{B_{-r} O}{\left(b_{r}+B_{-r}\right)^{2}}-1$ is strictly decreasing in $b_{r}$ given $O>0$ and $B_{-r}>0$, because $F$ is increasing, $\frac{b_{r} O}{b_{r}+B_{-r}}$ is increasing in $b_{r}$, and $\frac{B_{-r} O}{\left(b_{r}+B_{-r}\right)^{2}}$ is decreasing in $b_{r}$. Thus, $\pi_{r}$ is concave in $b_{r}$, and hence quasi-concave in $b_{r}$. Therefore, there exists a pure-strategy Nash equilibrium for the subgame of retailers, and letting $\theta_{r}$ be the Lagrange multiplier for constraint $b_{r} \geq 0$, this subgame can be characterized by the following first-order conditions:

$$
\begin{aligned}
& \frac{\partial \pi_{r}}{\partial b_{r}}=\left(p-(p-v) F\left(\frac{\check{b}_{r} O}{\check{B}}\right)\right) \frac{\left(\check{B}-\breve{b}_{r}\right) O}{(\check{B})^{2}}+\check{\theta}_{r}-1=0, \text { for all } r \in\{1,2, \ldots, R\}, \\
& \check{b}_{r} \geq 0, \check{\theta}_{r} \geq 0, \check{\theta}_{r} \breve{b}_{r}=0, \text { for all } r \in\{1,2, \ldots, R\} .
\end{aligned}
$$

Suppose to the contrary that retailers' equilibrium procurement budgets are asymmetric. Then, there must be at least two retailers (labeled as retailer 1 and 2 without loss of generality) such that $\breve{b}_{1}>\breve{b}_{2}$. Because $\breve{b}_{1}>\breve{b}_{2}$, we have $\breve{b}_{1}>0$, and hence $\breve{\theta}_{1}=0$ from (EC.30). Furthermore, as we showed earlier, $\partial \pi_{r} / \partial b_{r}$ is strictly decreasing in $b_{r}$, so $\left(p-(p-v) F\left(\frac{\breve{b}_{1} O}{\breve{B}}\right)\right) \frac{\left(\breve{B}-\breve{b}_{1}\right) O}{(\check{B})^{2}}-1<$ $\left(p-(p-v) F\left(\frac{\breve{b}_{2} O}{\breve{B}}\right)\right) \frac{\left(\breve{B}-\breve{b}_{2}\right) O}{(\check{B})^{2}}-1 \leq 0$. Thus, $\breve{b}_{1}$ cannot satisfy (EC.29), which is a contradiction. Thus, the equilibrium should be symmetric.

Given the total production quantity $O$, the retailer's unique symmetric best-response procurement budget satisfies:

$$
\breve{b}=\left[(p-v)\left(1-F\left(\frac{O}{R}\right)\right)+v\right] \frac{O(R-1)}{R^{2}}>0 .
$$

Second, each supplier $s$ 's action is his production quantity $o_{s} \in \mathbb{R}_{+}$, so his action set is $\mathbb{R}_{+}$. Furthermore, supplier s's revenue $w o_{s}=\frac{\breve{B} o_{s}}{o_{s}+O_{-s}} \leq \breve{B}$. Then, the benefit of $o_{s}$ to the supplier is bounded because as we discussed above, $\breve{b}_{r}$ is bounded. The cost of $o_{s}$ is unbounded, so there exists an upper bound $\bar{o}$ on the optimal production quantity. Without loss of optimality, the supplier's action set can be restricted to $[0, \bar{o}]$, which is compact and convex. By incorporating the retailer's equilibrium procurement budget to the supplier's profit, we get the supplier's objective in the Stackelberg game, which is to maximize

$\frac{o_{s}}{o_{s}+O_{-s}}\left[\left((p-v)\left(1-F\left(\frac{O}{R}\right)\right)+v\right) \frac{O(R-1)}{R}\right]-c o_{s}=o_{s}\left[\left((p-v)\left(1-F\left(\frac{O}{R}\right)\right)+v\right) \frac{(R-1)}{R}\right]-c o_{s}$.

The first derivative of the supplier's profit with respect to $o_{s}$ is

$$
\frac{\partial \pi_{s}}{\partial o_{s}}=(p-v)\left(1-F\left(\frac{O}{R}\right)\right) \frac{(R-1)}{R}-\left(o_{s}(p-v) f\left(\frac{O}{R}\right) \frac{(R-1)}{R^{2}}\right)+\frac{(R-1)}{R} v-c .
$$

This first derivative can be rewritten as

$$
\frac{\partial \pi_{s}}{\partial o_{s}}=\left(1-F\left(\frac{O}{R}\right)\right)\left[(p-v) \frac{(R-1)}{R}-\left(\frac{o_{s}}{O}(p-v) \frac{R-1}{R} \frac{\frac{O}{R} f\left(\frac{O}{R}\right)}{1-F\left(\frac{O}{R}\right)}\right)\right]+\frac{(R-1)}{R} v-c .
$$


Because $f$ has an increasing generalized failure rate, we have $\frac{f(x) x}{1-F(x)}$ increasing in $x$, which requires that $\frac{f(x) x}{1-F(x)}<+\infty$ for all $x$. Thus, under the assumption that $p>\frac{R}{R-1} c, \lim _{o_{s} \rightarrow 0} \frac{\partial \pi_{s}}{\partial o_{s}}>0$. Then, the supplier's solution should be interior. Finally, we show that the supplier profit $\pi_{s}$ is unimodal in $o_{s}$, which guarantees that the first-order conditions yield unique optimum. Let $\rho\left(o_{s} \mid O_{-s}\right) \equiv$ $(p-v) \frac{(R-1)}{R}-\left(\frac{o_{s}}{O}(p-v) \frac{R-1}{R} \frac{\frac{O}{R} f\left(\frac{O}{R}\right)}{1-F\left(\frac{O}{R}\right)}\right)$. Clearly, $\lim _{o_{s} \rightarrow 0} \rho\left(o_{s} \mid O_{-s}\right)=(p-v) \frac{(R-1)}{R}>0, \rho\left(o_{s} \mid O_{-s}\right)$ is continuous and decreasing in $o_{s}$, and $\lim _{o_{s} \rightarrow \infty} \rho\left(o_{s} \mid O_{-s}\right)=-\infty$ because by assumption, $\frac{f(x) x}{1-F(x)}$ is increasing and unbounded in $x$. Then, there exists $o^{\rho}$ such that $\rho\left(o_{s} \mid O_{-s}\right)>0$ for $o_{s}<o^{\rho}$ and $\rho\left(o_{s} \mid O_{-s}\right) \leq 0$ for $o_{s} \geq o^{\rho}$. For $o_{s}<o^{\rho}$, because both $\rho\left(o_{s} \mid O_{-s}\right)$ and $\left(1-F\left(o_{s}\right)\right)$ are positive and decreasing in $o_{s}$, we have $\partial \pi_{s} / \partial o_{s}$ decreasing in $o_{s}$. For $o_{s} \geq o^{\rho}, \partial \pi_{s} / \partial o_{s}<0$ because $\rho\left(o_{s} \mid O_{-s}\right) \leq 0$ and $v<c$. Furthermore, as discussed above, $\lim _{o_{s} \rightarrow 0} \frac{\partial \pi_{s}}{\partial o_{s}}>0$. Thus, $\pi_{s}$ is unimodal and there exists a unique $o_{s}$ such that $\partial \pi_{s} / \partial o_{s}=0$. Therefore, the following first-order conditions yield a unique solution to the supplier's profit maximization problem:

$$
\frac{\partial \pi_{s}}{\partial o_{s}}\left(\widehat{o}_{s}\right)=\left(1-F\left(\frac{\widehat{O}}{R}\right)\right) \rho\left(\widehat{o}_{s} \mid \widehat{O}_{-s}\right)+\frac{(R-1)}{R} v-c=0 .
$$

Suppose to the contrary that suppliers' equilibrium production quantities are asymmetric. Then, there must be at least two suppliers (labeled as supplier 1 and 2 without loss of generality) such that $\widehat{o}_{1}>\widehat{o}_{2}$. From the discussion above, we can see that $0<\widehat{o}_{2}<\widehat{o}_{1}<o^{\rho}$. Then, because $\partial \pi_{s} / \partial o_{s}$ is decreasing as we discussed above, we have $\frac{\partial \pi_{s}}{\partial o_{s}}\left(\widehat{o}_{2}\right)>\frac{\partial \pi_{s}}{\partial o_{s}}\left(\widehat{o}_{1}\right)$. Thus, $\widehat{o}_{1}$ or $\widehat{o}_{2}$ does not satisfy (EC.32). This contradicts the fact that both $\widehat{o}_{1}$ and $\widehat{o}_{2}$ are best-response production quantities. Therefore, the equilibrium is symmetric. Letting $\widehat{q}=\frac{\widehat{O}}{R}$, we have the following first-order condition:

$$
(1-F(\widehat{q}))\left[(p-v)-\left(\frac{1}{S}(p-v) \frac{\widehat{q} f(\widehat{q})}{(1-F(\widehat{q}))}\right)\right]+v-\frac{R}{R-1} c=0 .
$$

After simplifications, we obtain the following characterizing equation for $\widehat{q}$ :

$$
\Omega(\widehat{q}, R, S) \equiv F(\widehat{q})+f(\widehat{q}) \widehat{q} \frac{1}{S}-\frac{p-\frac{R}{R-1} c}{p-v}=0 .
$$

Given (EC.33), the equilibrium wholesale price satisfies

$$
\widehat{w}=\frac{R \widehat{b}}{\widehat{O}}=(p-(p-v) F(\widehat{q})) \frac{(R-1)}{R}=\left((p-v) f(\widehat{q}) \widehat{q} \frac{(R-1)}{S R}\right)+c .
$$

(b) If we apply the Implicit Function Theorem on (EC.33), we can see that $\frac{\partial \widehat{q}}{\partial S}=-\frac{\partial \Omega(\widehat{q}, R, S)}{\partial S} / \frac{\partial \Omega(\widehat{q}, R, S)}{\partial \widehat{q}}$ and $\frac{\partial \widehat{q}}{\partial R}=-\frac{\partial \Omega(\widehat{q}, R, S)}{\partial R} / \frac{\partial \Omega(\widehat{q}, R, S)}{\partial \widehat{q}}$. We have these derivatives as follows:

$$
\begin{aligned}
& \frac{\partial \Omega(\widehat{q}, R, S)}{\partial \widehat{q}}=f(\widehat{q})+f(\widehat{q}) \frac{1}{S}+f^{\prime}(\widehat{q}) \widehat{q} \frac{1}{S}, \\
& \frac{\partial \Omega(\widehat{q}, R, S)}{\partial S}=-f(\widehat{q}) \widehat{q} \frac{1}{S^{2}}<0, \\
& \frac{\partial \Omega(\widehat{q}, R, S)}{\partial R}=\frac{-1}{(R-1)^{2}} \frac{c}{p-v}<0 .
\end{aligned}
$$


Because $f$ has an increasing generalized failure rate, i.e., $\frac{f(x) x}{1-F(x)}$ is increasing in $x$, we have the derivative condition $f(x)+f^{\prime}(x) x+\frac{f(x)^{2} x}{1-F(x)}>0$. Since $F(\widehat{q})+f(\widehat{q}) \widehat{q} \frac{1}{S}=\frac{p-\frac{R}{R-1} c}{p-v}<1$, we have

$$
\begin{aligned}
\frac{\partial \Omega(\widehat{q}, R, S)}{\partial \widehat{q}} & =f(\widehat{q})+f(\widehat{q}) \frac{1}{S}+f^{\prime}(\widehat{q}) \widehat{q} \frac{1}{S}+\frac{f(\widehat{q})^{2} \widehat{q}}{S(1-F(\widehat{q}))}-\frac{f(\widehat{q})^{2} \widehat{q}}{S(1-F(\widehat{q}))} \\
& =\frac{1}{S}\left[f(\widehat{q})+f^{\prime}(\widehat{q}) \widehat{q}+\frac{f(\widehat{q})^{2} \widehat{q}}{(1-F(\widehat{q}))}\right]+f(\widehat{q})\left(1-\frac{f(\widehat{q}) \widehat{q}}{S(1-F(\widehat{q}))}\right)>0 .
\end{aligned}
$$

Therefore, $\frac{\partial \widehat{q}}{\partial S}>0$ and $\frac{\partial \widehat{q}}{\partial R}>0$.

Lemma EC.A3. (a) $\pi(q) \equiv(p-c) q-(p-v) E\left[\left(q-\mathcal{D}_{r}\right)^{+}\right]$is increasing in $q$ for all $q<q^{*}=$ $F^{-1}\left(\frac{p-c}{p-v}\right)$, and $\pi(q)$ is concave. (b) The supply chain profit $\widehat{\Pi}(\widehat{q})$ is increasing in the equilibrium order quantity $\widehat{q}$ for all $\widehat{q}<q^{*}$, and $\widehat{\Pi}(\widehat{q})$ is concave in $\widehat{q}$.

Proof. (a) The derivative of $\pi(q)$ with respect to $q$ is

$$
\pi^{\prime}(q)=(p-c)-(p-v) F(q)
$$

As $q^{*}=F^{-1}\left(\frac{p-c}{p-v}\right)$, we have $\pi^{\prime}\left(q^{*}\right)=(p-c)-(p-v) F\left(q^{*}\right)=0$. As $F$ is increasing, $\pi^{\prime}(q)=(p-c)-$ $(p-v) F(q)>(p-c)-(p-v) F\left(q^{*}\right)=0$ for $q<q^{*}=F^{-1}\left(\frac{p-c}{p-v}\right)$. As $\pi^{\prime}(q)>0$ for all $q<q^{*}, \pi(q)$ is increasing for all $q<q^{*}$. Also, as $\pi^{\prime}(q)$ in (EC.39) is decreasing in $q, \pi(q)$ is concave.

(b) The supply chain profit $\widehat{\Pi}=\sum_{r=1}^{R} \widehat{\pi}_{r}+\sum_{s=1}^{S} \widehat{\pi}_{s}$, where $\widehat{\pi}_{r}$ is the retailer profit and $\widehat{\pi}_{s}$ is the supplier profit. In equilibrium, the retailer profit is

$$
\widehat{\pi}_{r}=(p-\widehat{w}) \widehat{q}-(p-v) E\left[\left(\widehat{q}-\mathcal{D}_{r}\right)^{+}\right]
$$

Furthermore, plugging $\widehat{o}=R \widehat{q} / S$ into (EC.11) yields the equilibrium supplier profit as

$$
\widehat{\pi}_{s}=(\widehat{w}-c) \widehat{o}=(\widehat{w}-c) \frac{R \widehat{q}}{S} .
$$

Using $\widehat{\pi}_{r}$ in (EC.40) and $\widehat{\pi}_{s}$ in (EC.41), we calculate the supply chain profit $\widehat{\Pi}$ as:

$$
\begin{aligned}
\widehat{\Pi} & =R \widehat{\pi}_{r}+S \widehat{\pi}_{s}=R \widehat{q}(p-\widehat{w})-R(p-v) E\left[\left(\widehat{q}-\mathcal{D}_{r}\right)^{+}\right]+S(\widehat{w}-c) \frac{R \widehat{q}}{S} \\
& =R(p-c) \widehat{q}-R(p-v) E\left[\left(\widehat{q}-\mathcal{D}_{r}\right)^{+}\right]=R \pi(\widehat{q}) .
\end{aligned}
$$

Because $\pi^{\prime}(q)>0$ for all $q<q^{*}$, and $\widehat{q}<q^{*}$ as shown in Corollary EC.1, we have $\frac{\partial \widehat{\Pi}}{\partial q}(\widehat{q})=R \pi^{\prime}(\widehat{q})>0$ for all $\widehat{q}<q^{*}$, i.e., the supply chain profit $\widehat{\Pi}$ is increasing in $\widehat{q}$ for all $\widehat{q}<q^{*}$. Also, $\pi^{\prime}(q)$ in (EC.39) is decreasing, so $\widehat{\Pi}$ is concave in $\widehat{q}$.

Proof of Corollary $\boldsymbol{E}$ C.2. To calculate $E=\widehat{\Pi} / \Pi^{*}$, we first calculate the centralized supply chain profit $\Pi^{*}$ as:

$$
\Pi^{*}=R(p-c) q^{*}-R(p-v) E\left[\left(q^{*}-\mathcal{D}\right)^{+}\right]=R \pi\left(q^{*}\right) .
$$


By using $\widehat{\Pi}$ and $\Pi^{*}$, we calculate the supply chain efficiency $E$ as follows:

$$
E=\frac{\widehat{\Pi}}{\Pi^{*}}=\frac{\widehat{q}(p-c)-(p-v) E\left[\left(\widehat{q}-\mathcal{D}_{r}\right)^{+}\right]}{q^{*}(p-c)-(p-v) E\left[\left(q^{*}-\mathcal{D}_{r}\right)^{+}\right]}=\frac{\pi(\widehat{q})}{\pi\left(q^{*}\right)} .
$$

The derivative of $\widehat{\Pi}$ with respect to $S$ is $\frac{\partial \widehat{\Pi}}{\partial S}=\frac{\partial \widehat{\Pi}}{\partial \widehat{q}} \frac{\partial \widehat{q}}{\partial S}$ and with respect to $R$ is $\frac{\partial \widehat{\Pi}}{\partial R}=\frac{\partial \widehat{\Pi}}{\partial \widehat{q}} \frac{\partial \widehat{q}}{\partial R}$. As Corollary EC.1 shows, $\partial \widehat{q} / \partial S>0$ and $\partial \widehat{q} / \partial R>0$; and as Lemma EC.A3 shows, $\partial \widehat{\Pi} / \partial \widehat{q}>0$, so we have $\partial \widehat{\Pi} / \partial S>0$ and $\partial \widehat{\Pi} / \partial R>0$. As $\pi(q)$ increases with $q$ for all $q<q^{*}$ from Lemma EC.A3, as $\widehat{q}<q^{*}$ and $\widehat{q}$ increases with $S$ and $R$ from Corollary EC.1, and $q^{*}$ does not change with $S$ and $R$, the supply chain efficiency $E$ in (EC.44) increases with $S$ and $R$.

Proof of Corollary EC.3. Let $w(R, S)$ and $q(R, S)$ be two functions such that

$$
\begin{aligned}
& F(q(R, S))+f(q(R, S)) q(R, S) \frac{1}{S}-\frac{p-\frac{R}{R-1} c}{p-v}=0 \\
& w(R, S)=\left((p-v) f(q(R, S)) q(R, S) \frac{R-1}{S R}\right)+c .
\end{aligned}
$$

For SC-m $(m \in\{A, B\})$, given $R_{m}$ and $S_{m}$, we can use Corollary EC.1 to establish that the equilibrium wholesale price $\widetilde{w}_{m}=w\left(R_{m}, S_{m}\right)$ and order quantity $\widetilde{q}_{m}=q\left(R_{m}, S_{m}\right)$.

(a) For this proof, we need the supplier profit $\widetilde{\pi}_{s, m}\left(\widetilde{q}_{m}\right)$ and the retailer profit $\widetilde{\pi}_{r, m}\left(\widetilde{q}_{m}\right)$ in SC-m ( $m \in\{A, B\}$ ) before integration. Plugging $\widetilde{o}_{m}=R_{m} \widetilde{q}_{m} / S_{m}$ into (EC.41) yields the supplier profit in SC-m $(m \in\{A, B\})$ before integration as:

$$
\widetilde{\pi}_{s, m}\left(\widetilde{q}_{m}\right)=\left(\widetilde{w}_{m}-c\right) \widetilde{o}_{m}=\left(\widetilde{w}_{m}-c\right) \frac{R_{m} \widetilde{q}_{m}}{S_{m}} .
$$

Using (EC.40), we can write the retailer profit before integration as:

$$
\widetilde{\pi}_{r, m}\left(\widetilde{q}_{m}\right)=\left(p-\widetilde{w}_{m}\right) \widetilde{q}_{m}-(p-v) E\left[\left(\widetilde{q}_{m}-\mathcal{D}_{r}\right)^{+}\right]
$$

Let $\pi(q) \equiv(p-c) q-(p-v) E\left[\left(q-\mathcal{D}_{r}\right)^{+}\right]$. Using (EC.47) and (EC.48), we obtain the total profit of firms in SC-m $(m \in\{A, B\})$ before integration as:

$$
\begin{aligned}
\widetilde{\Pi}_{m}\left(\widetilde{q}_{m}\right) \equiv R_{m} \widetilde{\pi}_{r, m}\left(\widetilde{q}_{m}\right)+S_{m} \widetilde{\pi}_{s, m}\left(\widetilde{q}_{m}\right) & =R_{m}\left(p-\widetilde{w}_{m}\right) \widetilde{q}_{m}-R_{m}(p-v) E\left[\left(\widetilde{q}_{m}-\mathcal{D}_{r}\right)^{+}\right]+\left(\widetilde{w}_{m}-c\right) R_{m} \widetilde{q}_{m} \\
& =R_{m}\left[(p-c) \widetilde{q}_{m}-(p-v) E\left[\left(\widetilde{q}_{m}-\mathcal{D}_{r}\right)^{+}\right]\right]=R_{m} \pi\left(\widetilde{q}_{m}\right) . \quad \text { EC.49) }
\end{aligned}
$$

After supply chain integration, the total profit of firms in SC-m $(m \in\{A, B\})$ is

$$
\begin{aligned}
R_{m} \widehat{\pi}_{r}(\widehat{q})+S_{m} \widehat{\pi}_{s}(\widehat{q}) & =R_{m}(p-\widehat{w}) \widehat{q}-R_{m}(p-v) E\left[\left(\widehat{q}-\mathcal{D}_{r}\right)^{+}\right]+(\widehat{w}-c) \frac{S_{m} R \widehat{q}}{S}+R_{m} c \widehat{q}-R_{m} c \widehat{q} \\
& =R_{m} \pi(\widehat{q})-R_{m} \widehat{w} \widehat{q}+(\widehat{w}-c) \frac{S_{m} R \widehat{q}}{S}+R_{m} c \widehat{q} \\
& =R_{m} \pi(\widehat{q})+(\widehat{w}-c) \widehat{q}\left(\frac{S_{m} R}{S}-R_{m}\right) .
\end{aligned}
$$


SC-m $(m \in\{A, B\})$ benefits from supply chain integration when the total profit of firms in SC-m after integration is larger than that before integration, which means

$R_{m} \pi(\widehat{q})+(\widehat{w}-c) \widehat{q}\left(\frac{S_{m} R}{S}-R_{m}\right)>R_{m} \pi\left(\widetilde{q}_{m}\right) \Longleftrightarrow \frac{S_{m}}{S}>\frac{R_{m}}{R}\left(1-\frac{\left(\pi(q(R, S))-\pi\left(q\left(R_{m}, S_{m}\right)\right)\right.}{(w(R, S)-c) q(R, S)}\right)$.

(b) As $\widetilde{q}_{m}<\widehat{q}$ and $\widehat{q}<q^{*}$ from Corollary EC.1, we have $\widetilde{q}_{m}<\widehat{q}<q^{*}$. As $\pi(q)$ increases with $q$ for all $q<q^{*}$ from Lemma EC.A3, using (EC.49), we get $\widetilde{\Pi}_{m}\left(\widetilde{q}_{m}\right)=R_{m} \pi\left(\widetilde{q}_{m}\right)<R_{m} \pi(\widehat{q})$ for $m \in\{A, B\}$. Then, the sum of total profits of SC-A and SC-B is less than the profit of the integrated supply chain as:

$$
\widetilde{\Pi}_{A}\left(\widetilde{q}_{A}\right)+\widetilde{\Pi}_{B}\left(\widetilde{q}_{B}\right)<R_{A} \pi(\widehat{q})+R_{B} \pi(\widehat{q})=R \pi(\widehat{q})=\Pi(\widehat{q}) .
$$

Proof of Corollary EC.4. In a simultaneous-move market game, given the sum of other retailers' best-response procurement budgets $\breve{B}_{-r}$ and the sum of all suppliers' best-response production quantities $\check{O}$, each retailer $r$ chooses her procurement budget $b_{r}$ to maximize her expected profit

$$
\left(A-d \frac{b_{r} \check{O}}{b_{r}+\check{B}_{-r}}\right) \frac{b_{r} \check{O}}{b_{r}+\check{B}_{-r}}-b_{r} .
$$

The supplier's problem is as in (7). Evaluating the retailer's and supplier's first-order conditions at symmetric equilibrium yields the following simultaneous equations for the equilibrium procurement budget $\widehat{b}$ and production quantity $\widehat{o}$ :

$$
\begin{aligned}
& \left(A-2 d \frac{S \widehat{o}}{R}\right) \frac{(R-1) S \widehat{o}}{R^{2} \widehat{b}}=1, \\
& \frac{\widehat{b} R(S-1)}{S^{2} \widehat{o}}=c .
\end{aligned}
$$

By substituting $\widehat{q}=\frac{S}{R} \widehat{o}$ on (EC.52), we obtain the equilibrium order quantity $\widehat{q}=\left(\frac{A-\frac{c R S}{(R-1)(S-1)}}{2 d}\right)$. Thus, the equilibrium wholesale price $\widehat{w}=\frac{R \widehat{b}}{S \widehat{o}}=\frac{c S}{(S-1)}$. Since $\frac{R}{R-1}$ decreases with $R$ and $\frac{S}{S-1}$ decreases with $S, \widehat{q}$ increases with $R$ or $S$.

Proof of Corollary EC.5. (a) The derivative of $\widehat{\pi}_{r}$ with respect to $S$ is

$$
\frac{\partial \widehat{\pi}_{r}}{\partial S}=\frac{c}{(S-1)^{2}} \widehat{q}+\left(A-\frac{c S}{S-1}-2 d \widehat{q}\right) \frac{\partial \widehat{q}}{\partial S}=\frac{c}{(S-1)^{2}} \widehat{q}+\frac{c S}{(R-1)(S-1)} \frac{\partial \widehat{q}}{\partial S} .
$$

As Corollary EC.4 shows, $\partial \widehat{q} / \partial S>0$, so we get $\partial \widehat{\pi}_{r} / \partial S>0$. Thus, $\widehat{\pi}_{r}$ is increasing in $S$. The derivative of $\widehat{\pi}_{s}$ with respect to $S$ is

$$
\begin{aligned}
\frac{\partial \widehat{\pi}_{s}}{\partial S} & =\frac{c R(1-2 S)}{S^{2}(S-1)^{2}} \widehat{q}+\frac{c R}{S(S-1)} \frac{c R}{(R-1) 2 d(S-1)^{2}} \\
& =\frac{c R}{S(S-1)}\left(\frac{1-2 S}{S(S-1)} \widehat{q}+\frac{c R}{(R-1) 2 d(S-1)^{2}}\right) .
\end{aligned}
$$

We have $\partial \widehat{\pi}_{s} / \partial S>0$ if and only if $\frac{y}{(2 S-1)}>A-y$, where $y=\frac{c R S}{(R-1)(S-1)}$. After simplifications, this condition boils down to the one in the corollary. The rest of the proof follows similarly to the proof of Proposition 2. 
(b) The derivative of $\widehat{\pi}_{r}$ with respect to $R$ is

$$
\frac{\partial \widehat{\pi}_{r}}{\partial R}=\left(p-\frac{c S}{S-1}-2 d \widehat{q}\right) \frac{\partial \widehat{q}}{\partial R}=\left(-\frac{c S}{S-1}+\frac{c R S}{(R-1)(S-1)}\right) \frac{\partial \widehat{q}}{\partial R}=\frac{c S}{(R-1)(S-1)} \frac{\partial \widehat{q}}{\partial R} .
$$

As $\partial \widehat{q} / \partial R>0$ from Corollary EC.4, we have $\partial \widehat{\pi}_{r} / \partial R>0$, i.e., the retailer profit $\widehat{\pi}_{r}$ is increasing in $R$. As $\frac{c R}{S(S-1)}$ is increasing in $R$ and $\partial \widehat{q} / \partial R>0$, the supplier profit $\widehat{\pi}_{s}$ is increasing in $R$. The rest of the proof follows similarly to the proof of Proposition 2.

Proof of Corollary EC.6. For this proof, we use ${ }^{\wedge}$ (resp., $\sim$ ) to denote equilibrium outcomes in the integrated supply chain (resp., SC-A and SC-B). Let

$$
w(R, S)=\frac{c S}{S-1} \text { and } q(R, S)=\left(\frac{A-\frac{c R S}{(R-1)(S-1)}}{2 d}\right) .
$$

For SC-m $(m \in\{A, B\})$, given $R_{m}$ and $S_{m}$, we can use Corollary EC.4 to obtain the equilibrium wholesale price $\widetilde{w}_{m}=w\left(R_{m}, S_{m}\right)$ and order quantity $\widetilde{q}_{m}=q\left(R_{m}, S_{m}\right)$.

(a) Let $\pi(q) \equiv(A-d q-c) q$. We obtain the total profit of firms in SC-m $(m \in\{A, B\})$ before integration as $\widetilde{\Pi}_{m}\left(\widetilde{q}_{m}\right) \equiv R_{m} \widetilde{\pi}_{r, m}\left(\widetilde{q}_{m}\right)+S_{m} \widetilde{\pi}_{s, m}\left(\widetilde{q}_{m}\right)=R_{m} \pi\left(\widetilde{q}_{m}\right)$. After supply chain integration, the total profit of firms in SC-m $(m \in\{A, B\})$ is

$$
R_{m} \widehat{\pi}_{r}(\widehat{q})+S_{m} \widehat{\pi}_{s}(\widehat{q})=R_{m} \pi(\widehat{q})+\left(\frac{S_{m}}{S}-\frac{R_{m}}{R}\right) \frac{c R \widehat{q}}{S-1} .
$$

SC-m $(m \in\{A, B\})$ benefits from supply chain integration when the total profit of firms in SC-m after integration is larger than that before integration, which means

$$
R_{m} \pi(\widehat{q})+\left(\frac{S_{m}}{S}-\frac{R_{m}}{R}\right) \frac{c R \widehat{q}}{S-1}>R_{m} \pi\left(\widetilde{q}_{m}\right) \Longleftrightarrow \frac{S_{m}}{S}>\frac{R_{m}}{R}\left(1-\frac{(S-1)\left(\pi(q(R, S))-\pi\left(q\left(R_{m}, S_{m}\right)\right)\right.}{c q(R, S)}\right) .
$$

(b) As $\widetilde{q}_{m}<\widehat{q}$ and $\widehat{q}<q^{*}$ from Corollary EC.4, we have $\widetilde{q}_{m}<\widehat{q}<q^{*}$. As $\pi(q)$ increases with $q$ for all $q<q^{*}$, we get $\widetilde{\Pi}_{m}\left(\widetilde{q}_{m}\right)=R_{m} \pi\left(\widetilde{q}_{m}\right)<R_{m} \pi(\widehat{q})$ for $m \in\{A, B\}$. Then, the sum of total profits of SC-A and SC-B is less than the profit of the integrated supply chain as:

$$
\widetilde{\Pi}_{A}\left(\widetilde{q}_{A}\right)+\widetilde{\Pi}_{B}\left(\widetilde{q}_{B}\right)<R_{A} \pi(\widehat{q})+R_{B} \pi(\widehat{q})=R \pi(\widehat{q})=\Pi(\widehat{q}) .
$$

Proof of Corollary EC.7. In a simultaneous-move market game with demand uncertainty, given the sum of other retailers' best-response procurement budgets $\breve{B}_{-r}$ and the sum of all suppliers' best-response production quantities $\breve{O}$, each retailer $r$ chooses her procurement budget $b_{r}$ to maximize her expected profit

$$
p \frac{b_{r} \check{O}}{b_{r}+\check{B}_{-r}}-(p-v) E\left[\left(\frac{b_{r} \check{O}}{b_{r}+\check{B}_{-r}}-\mathcal{D}_{r}\right)^{+}\right]-b_{r} .
$$

The supplier's problem is as in (EC.11). Evaluating the retailer's and supplier's first-order conditions at symmetric equilibrium yields the following simultaneous equations for the equilibrium 
procurement budget $\widehat{b}$ and production quantity $\widehat{o}$ :

$$
\begin{aligned}
& \left(p-(p-v) F\left(\frac{S \widehat{o}}{R}\right)\right) \frac{(R-1) S \widehat{o}}{R^{2} \widehat{b}}=1, \\
& \frac{\widehat{b} R(S-1)}{S^{2} \widehat{o}}=c .
\end{aligned}
$$

By substituting $\widehat{q}=\frac{S}{R} \widehat{o}$ on (EC.58), we obtain the equilibrium order quantity $\widehat{q}=$ $F^{-1}\left(\frac{p-\frac{c R S}{(R-1)(S-1)}}{p-v}\right)$. Thus, the equilibrium wholesale price $\widehat{w}=\frac{R \widehat{b}}{S \widehat{o}}=\frac{c S}{(S-1)}$. Because $\frac{R S}{(R-1)(S-1)}>1$ and $F^{-1}$ is increasing, we have $\widehat{q}=F^{-1}\left(\frac{p-\frac{c R S}{(R-1)(S-1)}}{p-v}\right)<F^{-1}\left(\frac{p-c}{p-v}\right)=q^{*}$. Since $\frac{R}{R-1}$ decreases with $R$ and $\frac{S}{S-1}$ decreases with $S, \widehat{q}$ increases with $R$ or $S$.

Proof of Corollary EC.8. (a) The derivative of $\widehat{\pi}_{r}$ in (EC.40) with respect to $S$ is

$$
\frac{\partial \widehat{\pi}_{r}}{\partial S}=\frac{c}{(S-1)^{2}} \widehat{q}+\left(p-\frac{c S}{S-1}-(p-v) F(\widehat{q})\right) \frac{\partial \widehat{q}}{\partial S}=\frac{c}{(S-1)^{2}} \widehat{q}+\frac{c S}{(R-1)(S-1)} \frac{\partial \widehat{q}}{\partial S} .
$$

As Corollary EC.7 shows, $\partial \widehat{q} / \partial S>0$, so we get $\partial \widehat{\pi}_{r} / \partial S>0$. Thus, $\widehat{\pi}_{r}$ is increasing in $S$. The derivative of $\widehat{\pi}_{s}$ with respect to $S$ is

$$
\begin{aligned}
\frac{\partial \widehat{\pi}_{s}}{\partial S} & =\frac{c R(1-2 S)}{S^{2}(S-1)^{2}} \widehat{q}+\frac{c R}{S(S-1)} \frac{c R}{f(\widehat{q})(R-1)(p-v)(S-1)^{2}} \\
& =\frac{c R}{S(S-1)}\left(\frac{1-2 S}{S(S-1)} \widehat{q}+\frac{c R}{f(\widehat{q})(R-1)(p-v)(S-1)^{2}}\right) .
\end{aligned}
$$

We have $\partial \widehat{\pi}_{s} / \partial S>0$ if and only if $\frac{y}{(p-v)(2 S-1)}>F^{-1}\left(\frac{p-y}{p-v}\right) f\left(F^{-1}\left(\frac{p-y}{p-v}\right)\right)$, where $y=\frac{c R S}{(R-1)(S-1)}$.

Under the simultaneous-move game, the supply chain profit $\widehat{\Pi}$ is as in (EC.42), the centralized supply chain profit $\Pi^{*}$ is as in (EC.43), and the supply chain efficiency $E$ is as in (EC.44). The derivative of $\widehat{\Pi}$ with respect to $S$ is $\frac{\partial \widehat{\Pi}}{\partial S}=\frac{\partial \widehat{\Pi}}{\partial \widehat{q}} \frac{\partial \widehat{q}}{\partial S}$. As Corollary EC.7 shows, $\partial \widehat{q} / \partial S>0$; and as Lemma EC.A3 shows, $\partial \widehat{\Pi} / \partial \widehat{q}>0$, so we have $\partial \widehat{\Pi} / \partial S>0$. As $\pi(q)$ increases with $q$ for all $q<q^{*}$ from Lemma EC.A3, as $\widehat{q}<q^{*}$ and $\widehat{q}$ increases with $S$ from Corollary EC.7, and $q^{*}$ does not change with $S$, the supply chain efficiency $E$ increases with $S$.

(b) The derivative of $\widehat{\pi}_{r}$ in (EC.40) with respect to $R$ is

$$
\frac{\partial \widehat{\pi}_{r}}{\partial R}=\left(p-\frac{c S}{S-1}-(p-v) F(\widehat{q})\right) \frac{\partial \widehat{q}}{\partial R}=\left(-\frac{c S}{S-1}+\frac{c R S}{(R-1)(S-1)}\right) \frac{\partial \widehat{q}}{\partial R}=\frac{c S}{(R-1)(S-1)} \frac{\partial \widehat{q}}{\partial R} .
$$

As $\partial \widehat{q} / \partial R>0$ from Corollary EC.7, we have $\partial \widehat{\pi}_{r} / \partial R>0$, i.e., the retailer profit $\widehat{\pi}_{r}$ is increasing in $R$. As $\frac{c R}{S(S-1)}$ is increasing in $R$ and $\partial \widehat{q} / \partial R>0$, the supplier profit $\widehat{\pi}_{s}$ is increasing in $R$.

The derivative of $\widehat{\Pi}$ with respect to $R$ is $\frac{\partial \widehat{\Pi}}{\partial R}=\frac{\partial \widehat{\Pi}}{\partial \widehat{q}} \frac{\partial \widehat{q}}{\partial R}$. As $\partial \widehat{q} / \partial R>0$ from Corollary EC.7; and as Lemma EC.A3 shows, $\partial \widehat{\Pi} / \partial \widehat{q}>0$. Thus, we have $\partial \widehat{\Pi} / \partial R>0$. As $\pi(q)$ increases with $q$ for all $q<q^{*}$ from Lemma EC.A3, as $\widehat{q}<q^{*}$ and $\widehat{q}$ increases with $R$ from Corollary EC.7, and $q^{*}$ does not change with $R$, the efficiency $E$ increases with $R$. 
Proof of Corollary EC.9. For this proof, we use ${ }^{\wedge}$ (resp., $\sim$ ) to denote equilibrium outcomes in the integrated supply chain (resp., SC-A and SC-B). Let

$$
w(R, S)=\frac{c S}{S-1} \text { and } q(R, S)=F^{-1}\left(\frac{p-\frac{c R S}{(R-1)(S-1)}}{p-v}\right) .
$$

For SC-m ( $m \in\{A, B\}$ ), given $R_{m}$ and $S_{m}$, we can use Corollary EC.7 to obtain the equilibrium wholesale price $\widetilde{w}_{m}=w\left(R_{m}, S_{m}\right)$ and order quantity $\widetilde{q}_{m}=q\left(R_{m}, S_{m}\right)$.

(a) Let $\pi(q) \equiv(p-c) q-(p-v) E\left[\left(q-\mathcal{D}_{r}\right)^{+}\right]$. We obtain the total profit of firms in SC-m $(m \in$ $\{A, B\})$ before integration as $\widetilde{\Pi}_{m}\left(\widetilde{q}_{m}\right) \equiv R_{m} \widetilde{\pi}_{r, m}\left(\widetilde{q}_{m}\right)+S_{m} \widetilde{\pi}_{s, m}\left(\widetilde{q}_{m}\right)=R_{m} \pi\left(\widetilde{q}_{m}\right)$. After supply chain integration, the total profit of firms in $\mathrm{SC}-\mathrm{m}(m \in\{A, B\})$ is

$$
\begin{aligned}
R_{m} \widehat{\pi}_{r}(\widehat{q})+S_{m} \widehat{\pi}_{s}(\widehat{q}) & =R_{m}(p-\widehat{w}) \widehat{q}-R_{m}(p-v) E\left[\left(\widehat{q}-\mathcal{D}_{r}\right)^{+}\right]+(\widehat{w}-c) \frac{S_{m} R \widehat{q}}{S}+R_{m} c \widehat{q}-R_{m} c \widehat{q} \\
& =R_{m} \pi(\widehat{q})+(\widehat{w}-c) \widehat{q}\left(\frac{S_{m} R}{S}-R_{m}\right)=R_{m} \pi(\widehat{q})+\left(\frac{S_{m}}{S}-\frac{R_{m}}{R}\right) \frac{c R \widehat{q}}{S-1} .
\end{aligned}
$$

SC-m $(m \in\{A, B\})$ benefits from supply chain integration when the total profit of firms in SC-m after integration is larger than that before integration, which means

$R_{m} \pi(\widehat{q})+\left(\frac{S_{m}}{S}-\frac{R_{m}}{R}\right) \frac{c R \widehat{q}}{S-1}>R_{m} \pi\left(\widetilde{q}_{m}\right) \Longleftrightarrow \frac{S_{m}}{S}>\frac{R_{m}}{R}\left(1-\frac{(S-1)\left(\pi(q(R, S))-\pi\left(q\left(R_{m}, S_{m}\right)\right)\right.}{c q(R, S)}\right)$.

(b) As $\widetilde{q}_{m}<\widehat{q}$ and $\widehat{q}<q^{*}$ from Corollary EC.7, we have $\widetilde{q}_{m}<\widehat{q}<q^{*}$. As $\pi(q)$ increases with $q$ for all $q<q^{*}$ from Lemma EC.A3, we get $\widetilde{\Pi}_{m}\left(\widetilde{q}_{m}\right)=R_{m} \pi\left(\widetilde{q}_{m}\right)<R_{m} \pi(\widehat{q})$ for $m \in\{A, B\}$. Then, the sum of total profits of SC-A and SC-B is less than the profit of the integrated supply chain as:

$$
\widetilde{\Pi}_{A}\left(\widetilde{q}_{A}\right)+\widetilde{\Pi}_{B}\left(\widetilde{q}_{B}\right)<R_{A} \pi(\widehat{q})+R_{B} \pi(\widehat{q})=R \pi(\widehat{q})=\Pi(\widehat{q}) .
$$

\section{Additional References}

Cachon, G. P. 2004. Push, pull and advance-purchase discount contracts. Management Science 50(2) 222-238.

Cachon, G., C. Terwiesch. 2013. Matching supply with demand. McGraw-Hill International Edition, NY.

Dahan, E., H. Mendelson. 2001. An extreme-value model of concept testing. Management Science 47(1) 102-116.

EU. 2016. National accounts by 10 branches - aggregates at current prices. http://ec.europa.eu/ eurostat $/$ product? code $=$ namanace10c\&language $=$ en $\&$ mode $=$ view. AccessedonAugust1,2016 .

Fudenberg, D., J. Tirole. 1991. Game Theory. MIT Press, Cambridge, MA.

Gelman, A., J. B. Carlin, H. S. Stern, D. B. Rubin. 2003. Bayesian Data Analysis . Chapman \& Hall / CRC, Boca Raton, FL.

Lariviere, M. A., E. L. Porteus. 2001. Selling to the newsvendor: An analysis of price-only contracts. Manufacturing and Service Operations Management 3(4) 293-305. 